\title{
Structural properties and anion dynamics of yttrium dihydride and photochromic oxyhydride thin films examined by in situ $\mu^{+} \mathrm{SR}$
}

\author{
D. Chaykina $\odot,{ }^{1,2, *}$ T. de Krom $\odot,{ }^{2}$ G. Colombi $\odot,{ }^{1}$ H. Schreuders, ${ }^{1}$ A. Suter $\odot,{ }^{3}$ T. Prokscha $\odot,{ }^{3}$ B. Dam $\odot,{ }^{1}$ and S. Eijt $\odot^{2}$ \\ ${ }^{1}$ Materials for Energy Conversion and Storage, Department of Chemical Engineering, Delft University of Technology, \\ Van der Maasweg 9, NL-2629 HZ Delft, Netherlands \\ ${ }^{2}$ Fundamental Aspects of Materials and Energy, Department of Radiation Science and Technology, Faculty of Applied Sciences, \\ Delft University of Technology, Mekelweg 15, NL-2629 JB Delft, Netherlands \\ ${ }^{3}$ Laboratory for Muon Spin Spectroscopy, Paul Scherrer Institut, CH-5232 Villigen PSI, Switzerland
}

(Received 10 December 2020; accepted 4 June 2021; published 25 June 2021)

\begin{abstract}
Thin films of rare-earth metal oxyhydrides, such as yttrium oxyhydrides $\left(\mathrm{YH}_{3-2 x} \mathrm{O}_{x}\right)$, show a photochromic effect where the transparency of the films decreases reversibly upon exposure to UV light. However, the exact mechanism behind this effect is unknown. In this paper, we describe the behavior of $\mathrm{YH}_{3-2 x} \mathrm{O}_{x}$ thin films, with different $\mathrm{O}^{2-}: \mathrm{H}^{-}$ratios, under dark and illuminated conditions using in situ muon spin relaxation $\left(\mu^{+} \mathrm{SR}\right)$, and compare that to an oxygen-free reference compound, yttrium dihydride $\left(\mathrm{YH}_{2-\delta}\right)$. The muon acts as a local magnetic probe in our compounds, giving information related to electronic, structural, and photochromic properties. Although $\mathrm{YH}_{2-\delta}$ is the parent compound to $\mathrm{YH}_{3-2 x} \mathrm{O}_{x}$, the muon behavior in these two materials is different - the muon electrostatically interacts primarily with $\mathrm{H}^{-}$(dihydride) or $\mathrm{O}^{2-}$ (oxyhydride) - leading to the use of different theoretical models. For $\mathrm{YH}_{2-\delta}$, we observed the formation of an entangled $\mathrm{H}-\mu$ complex and the onset of $\mathrm{Mu}^{+}$diffusion and $\mathrm{H}^{-}$rearrangement above $150 \mathrm{~K}\left(E_{A, \Gamma}=67 \pm 13 \mathrm{meV}\right)$. For the oxyhydrides, we adopted a transition state model, where $\mathrm{Mu}^{0}$ formation and gradual $\mathrm{Mu}^{+}$recovery take place, accompanied by the formation of a $\mathrm{Mu}^{+}-\mathrm{O}^{2-}$ complex and a polaron at the $\mathrm{Y}$ cation. The activation energy $\left(E_{A, \text { dia }}\right)$ associated with $\mathrm{Mu}^{+}$recovery is dependent on lattice relaxation and is lower for thin films of higher $\mathrm{H}$ content $\left(E_{A, \mathrm{dia}}=29-45 \mathrm{meV}\right)$. In situ illumination further reduces this energy barrier for all measured oxyhydrides, suggesting that the photochromic effect involves a reversible structural rearrangement during photodarkening.
\end{abstract}

DOI: 10.1103/PhysRevB.103.224106

\section{INTRODUCTION}

Multianion compounds are a relatively new and emerging class of materials, containing two or more different anions [1]. While cation substitutions are more common, here, the variation of properties is achieved by anion substitution. Specifically, this study focuses on oxyhydrides, consisting of oxide $\left(\mathrm{O}^{2-}\right)$ and hydride $\left(\mathrm{H}^{-}\right)$anions.

Rare-earth metal (RE) oxyhydrides undergo a photochromic effect, and exhibit ionic conductivity as well. In particular, thin films of $R E \mathrm{H}_{3-2 x} \mathrm{O}_{x}$, where $R E=\mathrm{Sc}, \mathrm{Y}, \mathrm{Gd}$, Dy, Er [2-5], show the photochromic effect, while powdered $R E \mathrm{H}_{3-2 x} \mathrm{O}_{x}(R E=\mathrm{La}, \mathrm{Nd})[6,7]$ are reported as $\mathrm{H}^{-}$conductors. Because of this overlap, we suspect that photochromism and ionic mobility are related.

The photochromic effect is a phenomenon where the optical transmittance of a material changes when it is exposed to light with photon energy greater than its band gap. Thin

\section{*d.chaykina@tudelft.nl}

Published by the American Physical Society under the terms of the Creative Commons Attribution 4.0 International license. Further distribution of this work must maintain attribution to the author(s) and the published article's title, journal citation, and DOI. films of $R E \mathrm{H}_{3-2 x} \mathrm{O}_{x}$, for example, are transparent materials which become opaque under UV light [8]. The mechanism behind this color change in $R E \mathrm{H}_{3-2 x} \mathrm{O}_{x}$ is not well understood, but may follow a similar mechanism to $\mathrm{Cu}$-doped $\mathrm{Ag}$ halide glasses. When these glasses are exposed to light, electron-hole pairs are generated and atoms rearrange, creating metallic $\mathrm{Ag}^{0}$ clusters that account for the dark appearance $[9,10]$.

Similarly, $\mathrm{YH}_{3-2 x} \mathrm{O}_{x}$ shows increased conductivity [8] and disappearance of the most mobile $\mathrm{H}^{-}$fraction [11] under UV light, suggesting an analogous mechanism. However, there is an alternative explanation for photochromism which involves the presence of optically active defects (known as color centers) spanning the wide absorption range of these materials [9,12]. Further, although these materials may also be ion conductors, according to Ubukata et al. [7], certain $R E \mathrm{H}_{3-2 x} \mathrm{O}_{x}$ compounds are predicted to form anion-disordered compounds which would impede ionic mobility. Thus, any ion diffusion may be very local and not long-range.

To understand the photochromic effect, we investigated thin films of $\mathrm{YH}_{3-2 x} \mathrm{O}_{x}$ by low-energy muon spin rotation $\left(\mathrm{LE}-\mu^{+} \mathrm{SR}\right)$. These films are deposited by reactive magnetron sputtering $\mathrm{YH}_{x}$ at various deposition pressures $\left(p_{\mathrm{dep}}\right)$, and are then oxidized in ambient air [2]. If $p_{\text {dep }}<0.4 \mathrm{~Pa}$, the resultant film is metallic $\mathrm{YH}_{2-\delta}$. Transparent $\mathrm{YH}_{3-2 x} \mathrm{O}_{x}$ films are achieved when $p_{\text {dep }}>0.4 \mathrm{~Pa}$, resulting in increasing $\mathrm{O}^{2-}: \mathrm{H}^{-}$ratio on exposure to air as $p_{\text {dep }}$ increases. While 
$\mathrm{YH}_{2-\delta}$ films are nonphotochromic and metallic, the addition of oxygen changes the films to transparent, photochromic, and semiconducting $\mathrm{YH}_{3-2 x} \mathrm{O}_{x}$. The semiconducting gap is similar to that in the nonphotochromic $\mathrm{YH}_{3}$ phase. We showed previously that several properties evolve with the change in $\mathrm{O}^{2-}: \mathrm{H}^{-}$ratio, including the photochromic efficiency [5].

Here, we apply the muon spin rotation technique to study the electronic and structural properties of $\mathrm{YH}_{2-\delta}$ and $\mathrm{YH}_{3-2 x} \mathrm{O}_{x}$ thin films. The muon $\left(\mu^{+}\right)$is very sensitive to local magnetic fields which, in these compounds, are primarily due to the presence of $\mathrm{H}^{-}$ions which carry large nuclear magnetic dipole moments. This technique has been used to study ionic mobility in battery materials [13] and metal hydrides [14], as well as the excited configurations of $\mathrm{H}^{-}$in an oxyhydride $\left(\mathrm{BaTiO}_{3-x} \mathrm{H}_{x}\right)$ [15]. To investigate the photochromic effect, we use in situ $\mu^{+}$SR where the sample is illuminated to induce the photochromic effect during data collection.

In this paper, we show that $\mathrm{YH}_{2-\delta}$ and $\mathrm{YH}_{3-2 x} \mathrm{O}_{x}$ are distinctly different compounds. Local $\mathrm{H}^{-}$mobility and formation of a $\mathrm{H}-\mu$ spin-entangled complex was observed in sub-stoichiometric $\mathrm{YH}_{\sim 1.9}$. In the oxyhydrides, we used a transition state model [16] to explain the muon behavior. This model involves a temperature-driven change in the muon configuration from interstitial $\mathrm{Mu}^{0}$ (octahedral site) to bound $\mathrm{Mu}^{+}-\mathrm{O}^{2-}$. The transition is characterized by a lattice deformation and relaxation around $\mathrm{Mu}^{0}$, and the formation of a polaron on the $\mathrm{Y}^{3+}$ cation. The associated lattice deformation had a lower activation energy for H-rich oxyhydrides, and was further lowered by illumination. This suggests that a reversible change in the lattice occurs during photochromism, providing an element for the understanding of the photochromic mechanism in rare-earth metal oxyhydrides.

\section{EXPERIMENTAL METHODS}

Thin films of yttrium dihydride $\left(\mathrm{YH}_{2-\delta}\right)$ and yttrium oxyhydrides $\left(\mathrm{YH}_{3-2 x} \mathrm{O}_{x}\right)$ were deposited on $25 \times 25 \mathrm{~mm}$ quartz plates (Ted Pella, Inc.) by reactive magnetron sputtering as described in a previous publication [2]. The deposition pressure was kept constant by a flow of $\mathrm{Ar}$ and $\mathrm{H}_{2}$ gases at a ratio of $7: 1 \mathrm{Ar} / \mathrm{H}_{2} . \mathrm{YH}_{2-\delta}$ was deposited at a total pressure of $0.3 \mathrm{~Pa}$, while the oxyhydrides were deposited at $0.5,0.6$, and $0.7 \mathrm{~Pa}$. From this point on, they will be referred to as YHO-05, YHO-06, and YHO-07, respectively. Briefly, the deposition pressure influences the $\mathrm{O}^{2-}: \mathrm{H}^{-}$ratio in the resultant films after exposure to air, where a higher pressure leads to more oxidation and a higher $\mathrm{O}^{2-}: \mathrm{H}^{-}$ratio [5]. Deposition times were chosen so that all the films were $\sim 150 \mathrm{~nm}$ thick. The $\mathrm{YH}_{2-\delta}$ was capped with a thin layer of $\mathrm{Pd}(\sim 12 \mathrm{~nm})$ directly after deposition to prevent oxidation [17].

The band gap energies and photochromic properties of the films were measured optically (see Fig. S1 of the Supplemental Material (SM) [18]) using a custom-built optical fiber spectrometer where the light sources were a deuterium and a quartz tungsten halogen lamp (DH2000-BAL, Ocean Optics B.V.) with a $\mathrm{Si}$ array wavelength-dispersive spectrometer (HR4000, Ocean Optics B.V.). The optical transmission of the $\mathrm{YH}_{3-2 x} \mathrm{O}_{x}$ films was measured the day of deposition, 5 days after, and 10 days after to ensure that the air-oxidation process was complete (see SM [18], Fig. S2). Tauc plots were used
TABLE I. Summary of oxyhydride properties measured by optical methods. The indirect band gap $\left(E_{g}\right)$ values for samples 10 days after deposition are shown along with two parameters describing the photochromic performance (contrast and bleaching rate constant, $\tau_{B}$, at $295 \mathrm{~K}$ ).

\begin{tabular}{lccc}
\hline \hline Compound Name & $E_{g}(\mathrm{eV})$ & Contrast $(\%)$ & $\tau_{B}(\mathrm{~min})$ \\
\hline YHO-05 & $2.50 \pm 0.05$ & 21 & $41 \pm 8$ \\
YHO-06 & $2.55 \pm 0.04$ & 16 & $23 \pm 3$ \\
YHO-07 & $2.58 \pm 0.03$ & 10 & $12 \pm 2$ \\
\hline \hline
\end{tabular}

to determine the indirect band gap energies of the $\mathrm{YH}_{3-2 x} \mathrm{O}_{x}$ films (Table I and SM [18], Table SI).

The photochromic properties were probed by illuminating the $\mathrm{YH}_{3-2 x} \mathrm{O}_{x}$ films with a narrow-wavelength LED $(\lambda=$ $385 \mathrm{~nm}, I \sim 75 \mathrm{~mW} / \mathrm{cm}^{2}$ ) for $2 \mathrm{~h}$ and measuring the average transmittance $(\lambda=450-1000 \mathrm{~nm})$ with respect to time. After $2 \mathrm{~h}$, the LED was turned off and the bleaching process was measured for several hours. The resultant contrast and bleaching rate constants are shown in Table I. The contrast is the amount of color change which occurs during illumination, or the change in transmittance after $2 \mathrm{~h}$ compared to the initial value $[2,5]$. The return of original transparency after the LED is turned off is characterized by a bleaching rate constant, $\tau_{B}$, based on first-order kinetics [3,5]. All optical measurements were taken at room temperature $(\sim 295 \mathrm{~K})$.

Muon spin relaxation $\left(\mu^{+} \mathrm{SR}\right)$ experiments were done with the low-energy $\mu^{+} \mathrm{SR}$ spectrometer $\left(\mathrm{LE}-\mu^{+} \mathrm{SR}\right)$ at the $\mu \mathrm{E} 4$ beamline [19] of the Swiss Muon Source (Paul Scherrer Institut, Switzerland). Transverse field (TF) and zero field (ZF) configurations were used, where the applied magnetic field in the TF had a magnitude of $10 \mathrm{mT}$. The temperature was varied between $10-300 \mathrm{~K}$ for the TF and $10-260 \mathrm{~K}\left(\mathrm{YH}_{3-2 x} \mathrm{O}_{x}\right)$ or $10-300 \mathrm{~K}\left(\mathrm{YH}_{2-\delta}\right)$ for the $\mathrm{ZF}$ experiments. The implantation profile of the muon beam was simulated for various implantation energies using TrimSP [20,21], and the implantation energy of $13.5 \mathrm{keV}$ was chosen specifically to probe the center of the film. For in situ experiments, a bluepoint LED (HoenleGroup, $\lambda=365 \mathrm{~nm}$ ) was mounted along the muon beam direction. $\mathrm{YH}_{3-2 x} \mathrm{O}_{x}$ films were illuminated during the $\mu^{+} \mathrm{SR}$ measurements for $\sim 2 \mathrm{~h}$ at $T=50 \mathrm{~K}$ and allowed to bleach back to their original state for several hours.

\section{RESULTS}

\section{A. Yttrium dihydride, $\mathbf{Y H}_{2-\delta}$ \\ 1. Transverse field}

Transverse field (TF) measurements were performed for the entire set of samples to characterize their electronic properties and the distribution widths of their local magnetic fields $\left(\Delta B_{\text {local }}\right)$. In particular, we use this information to characterize the structure of metallic $\mathrm{YH}_{2-\delta}$ and the mobility of $\mathrm{H}^{-} / \mathrm{Mu}^{+}$ in its lattice. The $\mathrm{YH}_{2-\delta}$ data were fitted by a Gaussian cosine [22]:

$$
A(t)=A_{\text {dia }, \mathrm{TF}} \cos (2 \pi v t+\phi) e^{-\frac{1}{2} \sigma_{\mathrm{TF}}^{2} t^{2}},
$$

where $A_{\text {dia,TF }}$ is the diamagnetic asymmetry (decay asymmetry of the muons precessing at the Larmor frequency), $v$ is the 
(a)

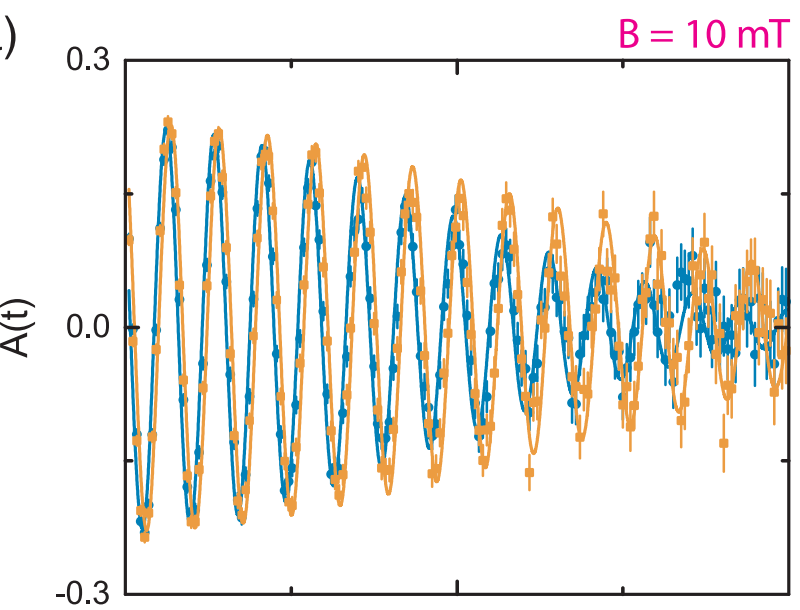

(b)

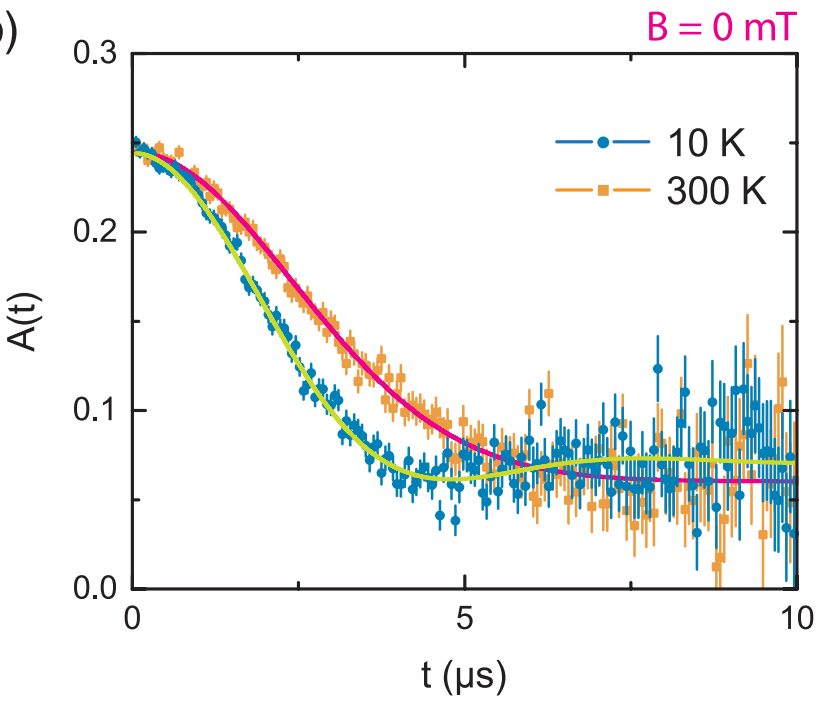

FIG. 1. $\mu^{+} \mathrm{SR}$ spectra for $\mathrm{YH}_{2-\delta}$ under (a) $\mathrm{TF}$ of $10 \mathrm{mT}$ and (b) ZF conditions. Measurements taken at $10 \mathrm{~K}$ and $300 \mathrm{~K}$ appear as blue and orange points, respectively. Solid curves correspond to best-fit analysis (see text).

Larmor frequency (related to external applied field, $B_{\text {ext }}$ ), and $\sigma_{\mathrm{TF}}$ is the Gaussian relaxation rate (related to $\Delta B_{\text {local }}$ ). More specifically, the relationship between $v$ and $B_{\text {ext }}$ is given by

$$
v=\frac{\gamma_{\mu} B_{\mathrm{ext}}}{2 \pi},
$$

where $\gamma_{\mu}$ is the muon gyromagnetic ratio $\left(\gamma_{\mu} / 2 \pi=\right.$ 135.53 MHz/T) [23]. Figure 1(a) shows fitted spectra of the time evolution of the muon spin polarization at the two extremes of temperature $(10$ and $300 \mathrm{~K})$. The frequency of the oscillations is related to the precession of the muon spin in the $B_{\text {ext }}$, described by the aforementioned Larmor frequency. The $A_{\mathrm{dia}, \mathrm{TF}}$ is obtained from the amplitude of the oscillations, while the $\sigma_{\mathrm{TF}}$ comes from the rate of decay of the cosine.

The electronic properties of our films are deduced from the asymmetry $\left(A_{\text {dia,TF }}\right)$ [Fig. 2(a)]. The asymmetry is close to the maximum values of the LE- $\mu^{+}$SR spectrometer and independent of temperature, consistent with the fact that $\mathrm{YH}_{2-\delta}$ is metallic. In general, implanted muons $\left(\mathrm{Mu}^{+}\right)$can interact with electrons during thermalization to form paramagnetic muonium $\left(\mathrm{Mu}^{0}\right)$ [24-26]. This $\mathrm{Mu}^{0}$ is normally considered

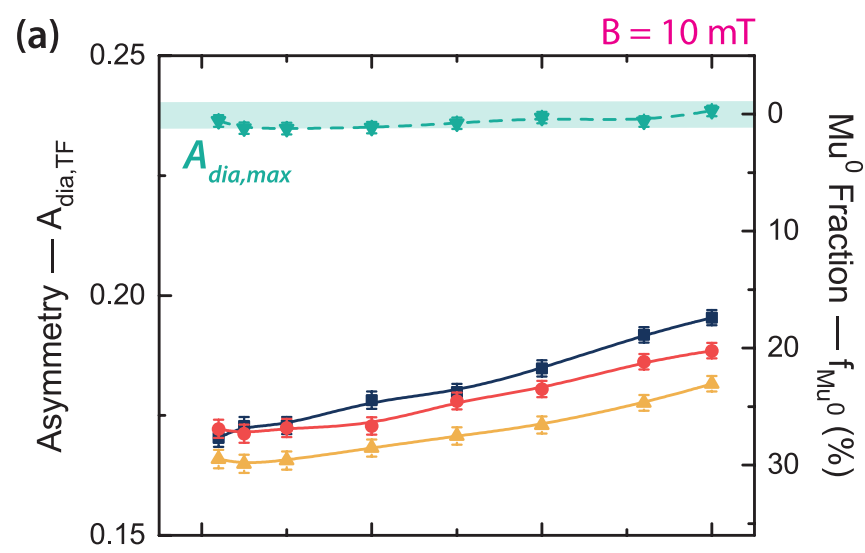

(b)

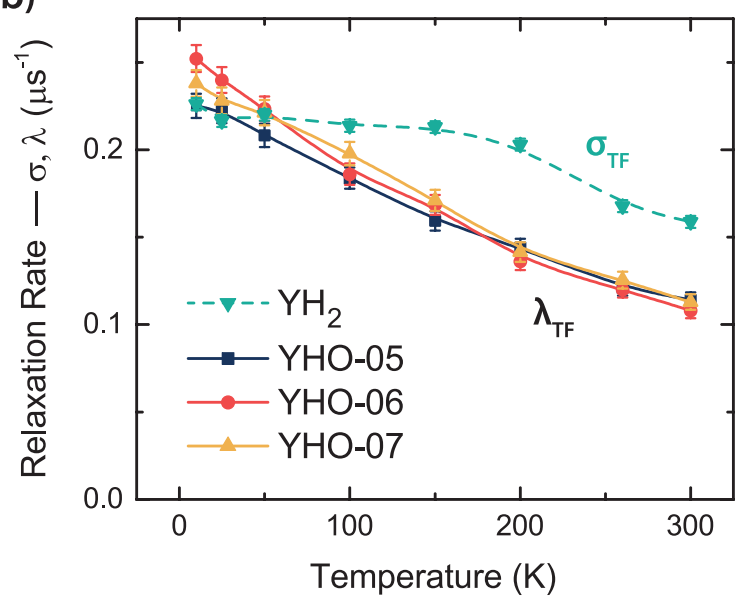

FIG. 2. Temperature dependence of the fitted (a) asymmetry and (b) relaxation rate from $\mathrm{TF}(B=10 \mathrm{mT})$ data of $\mathrm{YH}_{2-\delta}$ (green, dashed), YHO-05 (navy), YHO-06 (red), and YHO-07 (yellow). These were obtained from Eq. (1) for the $\mathrm{YH}_{2-\delta}$ and Eq. (8) for all the oxyhydrides. In (a), the asymmetry is converted to the $\mathrm{Mu}^{0}$ fraction based on $A_{\mathrm{dia}, \max }(0.2361 \pm 0.0012)$ by $f_{\mathrm{Mu}^{0}}=\left[A_{\mathrm{dia}, \max }-\right.$ $\left.A_{\text {dia, TF }}(T)\right] / A_{\text {dia,max }}$. Dashed and solid curves are guides to the eye.

a "missing fraction" of the asymmetry because its fast precession and depolarization make it difficult to observe in most $\mu^{+} \mathrm{SR}$ experiments [27]; thus, formation of $\mathrm{Mu}^{0}$ results in a reduced diamagnetic asymmetry. However, in metals, $\mathrm{Mu}^{0}$ formation is prevented due to electron shielding of the positive muon [23]. In $\mathrm{YH}_{2-\delta}$, we determine the maximum asymmetry attainable in our experiments, $A_{\text {dia, } \max }=0.2361 \pm 0.0012$. This is similar to other studies carried out at the same instrument [20] and acts as a reference point to characterize the oxyhydrides, discussed later.

The magnitude of $\sigma_{\mathrm{TF}}\left(\sim 0.22 \mu \mathrm{s}^{-1}\right)$ at low temperatures $(T<150 \mathrm{~K})$, shown in Fig. 2(b), is a clear indication that the local field distribution experienced by the $\mathrm{Mu}^{+}$is related to the nuclear dipolar fields of the surrounding nuclei, and similar values were found in other metal hydrides [14,28]. The nuclear dipolar fields are primarily the result of neighboring $\mathrm{H}^{-}$anions with a minor contribution from $\mathrm{Y}^{2+}$ cations. This is due to the large magnetic moment of hydrogen and negligible magnetic moment of yttrium in their most abundant isotopes $\left({ }^{1} \mathrm{H}=2.79 \mu_{N},{ }^{89} \mathrm{Y}=0.14 \mu_{N}\right)$. The hydrogen nuclei surrounding the muon have random spin directions, generating 


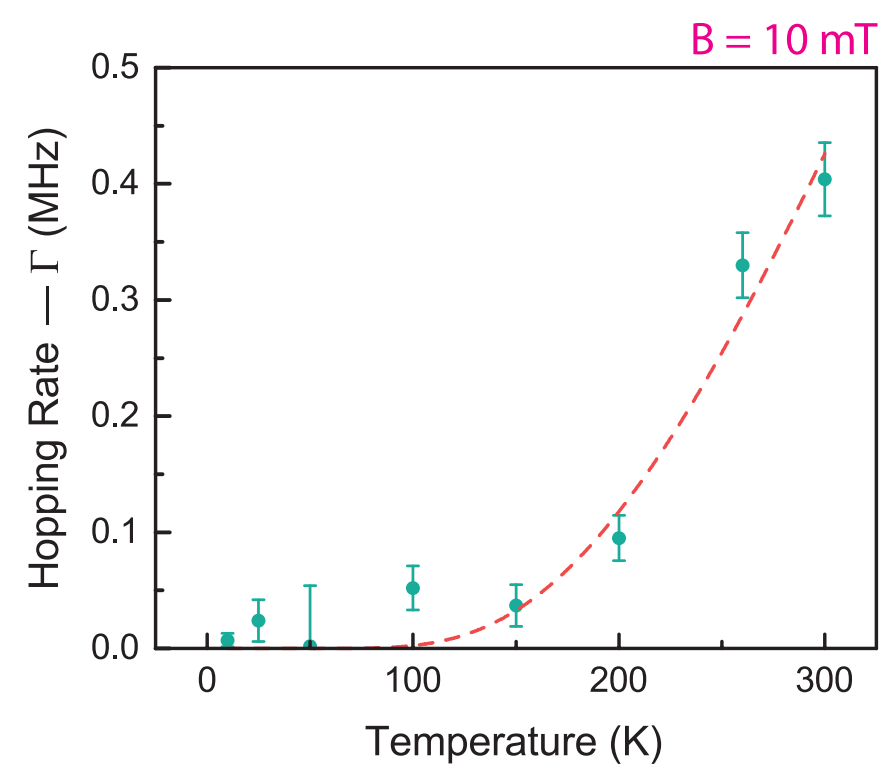

FIG. 3. The points indicate the hopping rate, $\Gamma$, obtained from fitting $\mathrm{YH}_{2-\delta} \mathrm{TF}$ data by the Abragam function, Eq. (3). These were further fitted by an Arrhenius equation (dashed line). The activation energy derived from this plot reflects the energy barrier for $\mathrm{Mu}^{+} / \mathrm{H}^{-}$ hopping in $\mathrm{YH}_{2-\delta}$ thin films.

a distribution of local magnetic fields with a Gaussian shape. Each muon sees a slightly different set of randomly oriented nuclear spins, resulting in the total muon depolarization observed in the experiment. Thus, $\sigma_{\mathrm{TF}}$ can be used to study the behavior of $\mathrm{H}^{-}$in $\mathrm{YH}_{2-\delta}$.

At low temperatures $(T=10-150 \mathrm{~K}), \sigma_{\mathrm{TF}}$ is constant [Fig. 2(b)], suggesting that the distribution of local magnetic fields, $\Delta B_{\text {local }}$, is static $\left(d \Delta B_{\text {local }} / d t=0\right)$ with respect to the muon lifetime $(2.2 \mu \mathrm{s})$. Above $150 \mathrm{~K}, \sigma_{\mathrm{TF}}$ decreases with increasing temperature, a clear sign of the onset of dynamic behavior known as "motional narrowing." If the $\mathrm{Mu}^{+}$or the nuclear magnetic moments around it start to move, the $B_{\text {local }}$ distribution will appear effectively narrower and the relaxation rate will decrease as a result. Potentially, both the $\mathrm{Mu}^{+}$ and the surrounding $\mathrm{H}^{-}$ions begin diffusing above $150 \mathrm{~K}$. Because it is not possible to differentiate between $\mathrm{Mu}^{+}$and $\mathrm{H}^{-}$diffusion by $\mu^{+} \mathrm{SR}$ [29], we assume at this point that both are mobile.

The Abragam function can help to further characterize the "motional narrowing" effect in $\mathrm{YH}_{2-\delta}[22,30]$ :

$$
\begin{aligned}
A(t)= & A_{\mathrm{TF}, \mathrm{dia}} \exp \left[-\frac{\sigma^{2} a b g}{\Gamma^{2}}[\exp (-\Gamma t)-1+\Gamma t]\right] \\
& \times \cos (2 \pi v t+\phi)
\end{aligned}
$$

This is a general function describing dynamics which considers a Gaussian relaxation $\left(\sigma_{a b g}\right)$ with the addition of a hopping rate $(\Gamma)$. When fitting the data, $\sigma_{a b g}$ was fixed to the low-temperature value of $\sigma_{\mathrm{TF}}$ from Fig. 2(b) [31,32]. As seen in Fig. $3, \Gamma$ is nearly zero between $10-150 \mathrm{~K}$, confirming that no dynamic behavior occurs in this temperature range. Above $150 \mathrm{~K}, \Gamma$ is nonzero and constantly increasing, indicating the onset of diffusion since the hopping rates, particularly above $200 \mathrm{~K}$, are larger than $\sigma_{a b g} \sim 0.2 \mathrm{MHz}[22,30]$.
The temperature dependency of the hopping rate is fitted by an Arrhenius equation (dashed line, Fig. 3):

$$
\Gamma=\Gamma_{o} \exp \left(-\frac{E_{A, \Gamma}}{k_{B} T}\right),
$$

where $\Gamma_{o}$ is the pre-exponential factor or attempt frequency, $E_{A, \Gamma}$ is the activation energy, and $k_{B}$ is the Boltzmann constant. The $E_{A, \Gamma}$ for $\mathrm{Mu}^{+} / \mathrm{H}^{-}$diffusion here is $770 \pm 150 \mathrm{~K}(67 \pm 13 \mathrm{meV})$ with a $\Gamma_{o}$ of $5.6 \pm$ 3.0 MHz. The interpretation of this is discussed in Sec. III A 3.

\section{Zero field}

The interaction between the muon spin and the sample environment under zero field $(\mathrm{ZF})$ conditions is different from what was observed under TF. In the latter case, the applied $B_{\text {ext }}$ is often larger than the strength of the interactions with the surroundings, so the muon spin simply precesses as $\mathrm{Mu}^{+}$ with a depolarization rate determined by the field distribution of the surrounding nuclear magnetic dipoles. In the absence of $B_{\text {ext }}$, the $\mathrm{Mu}^{+}$can, for example, form a spin-entangled complex with neighboring nuclei. This is particularly relevant for systems containing atoms with large nuclear magnetic moments (such as $\mathrm{H}^{-}$and $\mathrm{F}^{-}$), and has been used to describe muons in metal hydrides [14] and oxyhydrides [15]. We use the ZF depolarization signal of the complex to further analyze the structure of $\mathrm{YH}_{2-\delta}$ and the mobility of $\mathrm{H}^{-} / \mathrm{Mu}^{+}$.

The static and dynamic Gaussian Kubo-Toyabe (KT) functions were also considered for analyzing the $\mathrm{ZF}$ data, detailed in the Supplemental Material (see SM [18], Tables SII-SV). The static-KT analysis was disregarded due to a lower fit quality. The dynamic-KT analysis leads to a fit quality comparable to that of the $\mathrm{H}-\mu$ complex, but with significantly higher relaxation rates compared to the TF results, suggesting that this model is not compatible with the data.

The ZF spin depolarization of the $\mathrm{H}-\mu$ complex considered here is described by the following general expression:

$$
A(t)=F_{\mathrm{H} \mu}\left(A_{\mathrm{H} \mu}, \sigma_{\mathrm{ZF}}, f_{\mathrm{H} \mu}\right)+A_{\mathrm{NR}},
$$

where $A_{\mathrm{NR}}$ represents the fraction of nonrelaxing muons which do not form the $\mathrm{H}-\mu$ state, and $F_{\mathrm{H} \mu}$ is an equation describing a spin-entangled $\mathrm{H}-\mu$ complex $[15,33]$ :

$$
\begin{aligned}
F_{\mathrm{H} \mu}= & A_{\mathrm{H} \mu}\left[\frac{1}{6}+\frac{1}{6} \cos \left(2 \pi f_{\mathrm{H} \mu} t\right)+\frac{1}{3} \cos \left(\pi f_{\mathrm{H} \mu} t\right)\right. \\
& \left.+\frac{1}{3} \cos \left(3 \pi f_{\mathrm{H} \mu} t\right)\right] e^{-\frac{1}{2} \sigma_{\mathrm{ZF}}^{2} t^{2}} .
\end{aligned}
$$

In this equation, $A_{\mathrm{H} \mu}$ is the asymmetry related to the $\mathrm{H}-\mu$ complex, $\sigma_{\mathrm{ZF}}$ is the Gaussian relaxation rate due to surrounding nuclei, and $f_{\mathrm{H} \mu}$ is a frequency which depends on the distance between $\mathrm{H}^{-}$and $\mathrm{Mu}^{+}$as $[15,34]$

$$
f_{\mathrm{H} \mu}=\frac{\mu_{0} \hbar \gamma_{\mu} \gamma_{\mathrm{H}}}{8 \pi^{2} d^{3}} .
$$

Here, $\gamma_{\mu}$ and $\gamma_{\mathrm{H}}$ are the gyromagnetic ratios of a muon and a hydrogen atom, and $d$ is the $\mathrm{H}-\mu$ distance. An $\mathrm{H}-\mu$-H complex is also possible and has been used in highly concentrated systems [33-35]. However, we dismiss it here because it did not match the oscillations in our data [Fig. 1(b)], likely due to 


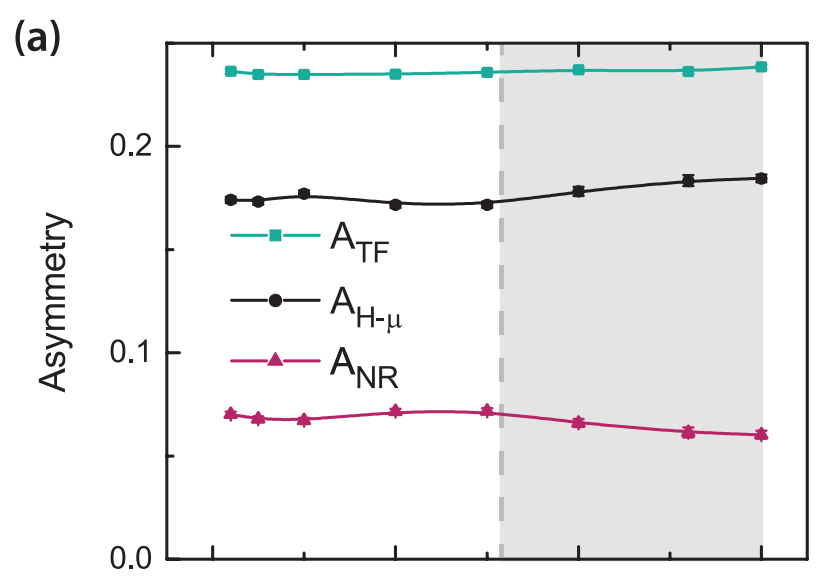

(b)

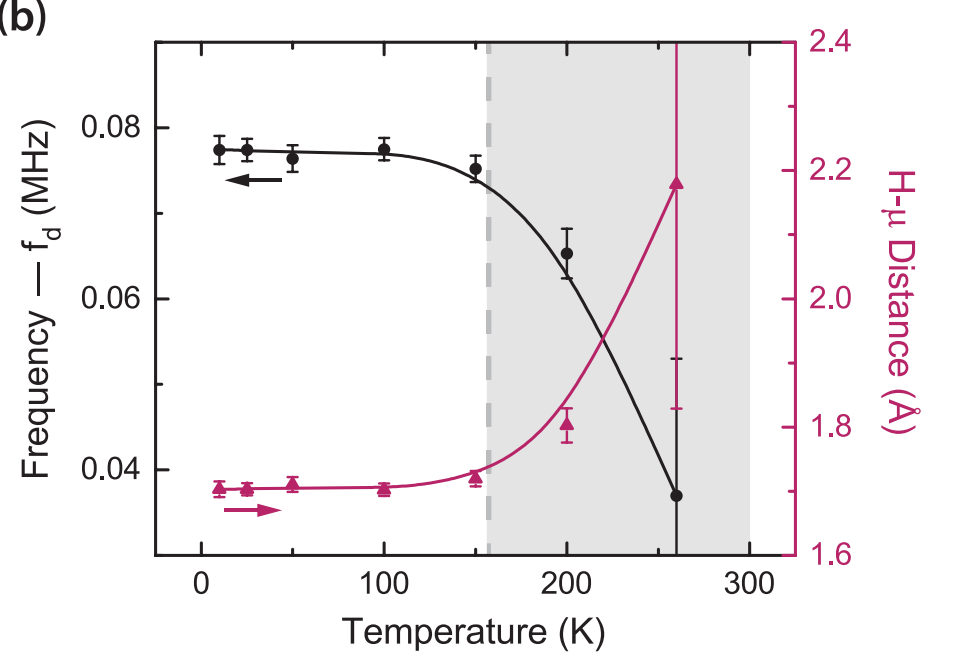

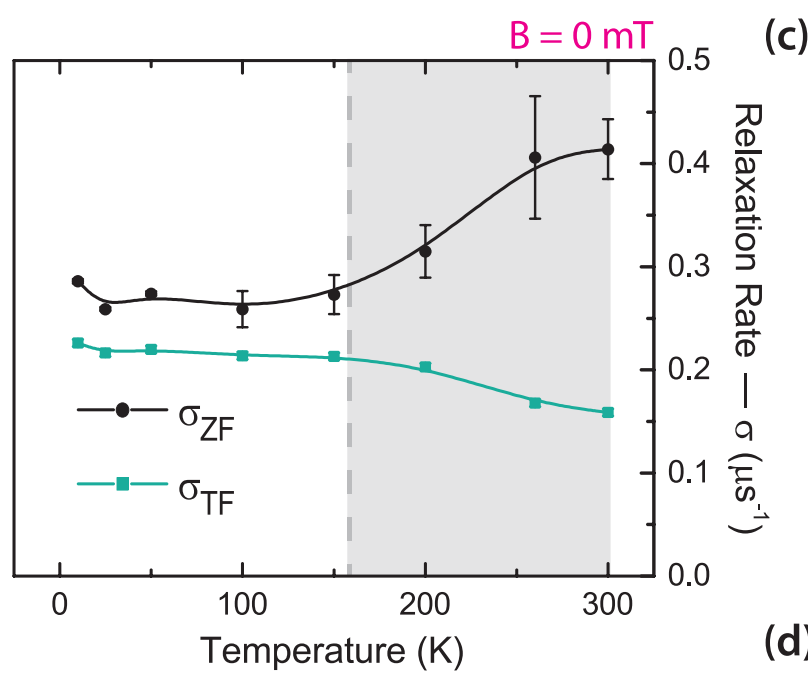

(d)

FIG. 4. Summary of parameters fitted from the H- $\mu$ equation. The gray separation denotes the static-to-dynamic transition found from TF, with mobility beginning above $150 \mathrm{~K}$. (a) The ZF asymmetry contributions are compared to the asymmetry found from TF. The sum of the two $\mathrm{ZF}$ contributions $\left(A_{\mathrm{H} \mu}+A_{\mathrm{NR}}\right)$ is equivalent to $A_{\mathrm{TF}, \text { dia }}$. (b) The fitted frequency (black) and calculated $\mathrm{H}-\mu$ distance (purple). (c) Comparison of the Gaussian relaxation rates from TF (green) and ZF (black). (d) Image of the proposed muon stopping site in $\mathrm{YH}_{2-\delta}$ based on the $\mathrm{H}-\mu$ equation fitting (see text).

the structural properties of $\mathrm{YH}_{2-\delta}$ involving the presence of anion vacancies, as discussed below.

The asymmetry contributions are plotted in Fig. 4(a). $A_{\mathrm{H} \mu}$ accounts for the majority of the total asymmetry and is independent of temperature. Since we observe this even above the static-to-dynamic transition temperature of $\sim 150 \mathrm{~K}$, we infer that the majority of $\mathrm{Mu}^{+}$are in the $\mathrm{H}-\mu$ complex for the entire temperature range $(10-300 \mathrm{~K})$. It is important to note that the total asymmetry $\left(A_{\mathrm{H} \mu}+A_{\mathrm{NR}}\right)$ is equivalent to the asymmetry found in the TF experiments $\left(A_{\mathrm{TF}}\right)$, validating the use of Eq. (5).

The frequency, $f_{\mathrm{H} \mu}$, and the corresponding $\mathrm{H}-\mu$ distance are plotted in Fig. 4(b). Up to $150 \mathrm{~K}$, the $\mathrm{H}-\mu$ distance is constant with a value of $1.71 \pm 0.01 \AA$. From this value, we propose a muon stopping site [Fig. 4(d)] where $\mathrm{Mu}^{+}$(the option $\mathrm{Mu}^{-}$is unlikely for the $\mathrm{H}-\mu$ complex in $\mathrm{YH}_{1.9}$, as explained in [36]) is placed next to a $\mathrm{H}^{-}$ion, and adjacent to an anion vacancy. We choose this site for several reasons. First, the distance between two anion sites is much larger than the $\mathrm{H}-\mu$ distance, so the $\mathrm{Mu}^{+}$cannot be in a nearest-neighbor anion position (tetrahedral to tetrahedral $\sim 2.75 \AA$, to octahe- dral $\sim 2.38 \AA$ ). Second, the equation for an entangled $\mathrm{H}-\mu$-H (see, e.g., Ref. [33]) complex did not fit the data, suggesting that the $\mathrm{Mu}^{+}$is not surrounded by multiple equidistant $\mathrm{H}^{-}$ ions. Lastly, an anion vacancy in this compound should be positively charged, slightly repelling the $\mathrm{Mu}^{+}$and pushing it toward the neighboring $\mathrm{H}^{-}$ion electrostatically.

Elongation of this $\mathrm{H}-\mu$ "bond" is observed for $T>150 \mathrm{~K}$; however, the error bars are very large as the frequency becomes poorly defined at high temperatures, visible also in the spectrum in Fig. 1(b). This elongation can be the result of thermal vibrations [33] or $\mathrm{Mu}^{+} / \mathrm{H}^{-}$hopping [14]. For $T \sim 200 \mathrm{~K}$, thermal vibrations are likely dominant since the hopping rate from TF was also low. However, for temperatures above $260 \mathrm{~K}$, the frequency becomes ill defined due to the gradual "breaking" of the $\mathrm{H}-\mu$ bond as the two species move away from each other in favor of mobility.

The Gaussian relaxation rate, $\sigma_{\mathrm{ZF}}$, is compared to $\sigma_{\mathrm{TF}}$ in Fig. 4(c), with $\sigma_{\mathrm{ZF}}$ coming from Eq. (6) and $\sigma_{\mathrm{TF}}$ from Eq. (1). Both relaxation rates are temperature-independent in the range of $10-150 \mathrm{~K}$, indicating static behavior. The value of $\sigma_{\mathrm{ZF}}$ is larger than $\sigma_{\mathrm{TF}}$ because in the TF, only the magnetic 
field distribution along the direction of $B_{\text {ext }}$ is measured, while in the ZF case, the muon is sensitive to the field distribution in all directions [22]. This results in $\sigma_{\mathrm{ZF}}>\sigma_{\mathrm{TF}}$.

Above $150 \mathrm{~K}, \sigma_{\mathrm{TF}}$ decreases while $\sigma_{\mathrm{ZF}}$ increases, both of which are indicators of $\mathrm{Mu}^{+} / \mathrm{H}^{-}$diffusion. The different temperature trends are a result of the different muon-sample spin interactions. Under TF conditions, the spin entanglement between $\mathrm{Mu}^{+}$and $\mathrm{H}^{-}$is quenched, and the muon is sensitive to the field distribution of the nuclear magnetic dipoles only. When the $\mathrm{Mu}^{+}$or $\mathrm{H}^{-}$begins to move, this field distribution is "motionally narrowed," as mentioned before, and the relaxation rate decreases. In the $\mathrm{ZF}$, however, the situation is different because the muon is spin-entangled to a neighboring $\mathrm{H}^{-}$. Mobility of the $\mathrm{Mu}^{+}$and $\mathrm{H}^{-}$leads to faster relaxation of the muon spin in this complex, thus a higher relaxation rate [33]. This finding confirms that $\mathrm{Mu}^{+}$and possibly $\mathrm{H}^{-}$, as discussed below, are mobile above $150-200 \mathrm{~K}$ in our $\mathrm{YH}_{2-\delta}$ films.

\section{Implications for the structure and $\mathrm{H}^{-}$dynamics of $\mathrm{YH}_{2-\delta}$}

A $\mathrm{YH}_{2-\delta}$ thin film is used here as a reference to study the behavior of $\mathrm{YH}_{3-2 x} \mathrm{O}_{x}$ thin films, which are made by airoxidation of $\mathrm{YH}_{2-\delta}$. Based on the temperature-independent asymmetries in both $\mathrm{TF}$ and $\mathrm{ZF}$, we conclude that no $\mathrm{Mu}^{0}$ formation occurred in the $\mathrm{YH}_{2-\delta}$ film, in accordance with its metallic behavior. Although Y-metal hydrides $\left(\mathrm{YH}_{x}\right)$ span a wide range of hydrogen concentration $(0 \leqslant x \leqslant 3)$, only compositions where $x$ approaches 3 are insulating, while the rest are metallic [17,37]. To obtain the $x \sim 3$ semiconducting state would require $\mathrm{H}_{2}(\mathrm{~g})$ partial deposition pressures much higher $\left(p_{\mathrm{H}_{2}} \geqslant 10 \mathrm{~Pa}\right)$ than what is used in our deposition procedure $[2,38]$. The formation of the dihydride phase requires only $p_{\mathrm{H}_{2}}>10^{-29} \mathrm{~Pa}[38]$.

Previous work has shown that the solubility of hydrogen in the dihydride phase ranges between sub-stoichiometric $\mathrm{YH}_{1.9}$ and super-stoichiometric $\mathrm{YH}_{2.1}$ [39]. These extremes can be distinguished based on their optical properties, where $\mathrm{YH}_{1.9}$ shows an optical transmission window, while $\mathrm{YH}_{2.1}$ is opaque. Optical measurements show a transmission window (see SM [18], Fig. S1), suggesting that our film has a composition of $\mathrm{YH}_{\sim 1.9}$. In such a compound there will be many anion vacancies due to the partial occupation of tetrahedral interstitial sites by $\mathrm{H}^{-}$. These structural characteristics lead to the assignment of the muon site in Fig. 4(d), explained in Sec. III A 2. DFT calculations could be helpful in order to examine in detail the muon site and energetics of muon binding in the $\mathrm{H}-\mu$ complex in the sub-stoichiometric $\mathrm{YH}_{1.9}$ phase, but are also challenging in view of disorder that may be present in the $\mathrm{H}^{-}$ anion sublattice.

We observe dynamic behavior in $\mathrm{YH}_{\sim 1.9}$ above $150 \mathrm{~K}$, which we attribute to $\mathrm{Mu}^{+}$and $\mathrm{H}^{-}$mobility. The activation energy $E_{A, \Gamma}$ we derived for this mobility $(67 \pm 13 \mathrm{meV})$ is much lower than the values obtained in other metal hydrides (e.g., $\mathrm{TiH}_{2 \pm \delta}$ and $\mathrm{YH}_{2 \pm \delta}$ ) for long-range $\mathrm{H}^{-}$motion $(\sim 400-500 \mathrm{meV})[28,40-43]$. It can instead be related to local anion reorganization. Particularly, Kossler et al. [28] found two "motional narrowing" steps in $\mathrm{YH}_{2.00}$, with the first relating to anion reorganization $\left(\mathrm{H}^{-}\right.$moving from tetrahedral to octahedral sites) and the second relating to long-range
$\mathrm{H}^{-}$diffusion. Additionally, a similar onset temperature was reported in $\mathrm{YH}_{2.1}$ where, at $155 \mathrm{~K}$, the resistivity changed due to the reorganization of $\mathrm{H}^{-}$anions in the octahedral sites, many of which are vacant [44]. Therefore, it is likely that in this $\mathrm{YH}_{\sim 1.9}$ thin film there is also a local rearrangement of $\mathrm{H}^{-}$ and vacancies above $\sim 150 \mathrm{~K}$.

The activation energy $E_{A, \Gamma}$ obtained here is likely influenced by the simultaneous diffusion of $\mathrm{Mu}^{+}$throughout the material. $\mathrm{Mu}^{+}$diffusion was reported in several other fcc metals with comparable activation energies [31]. Not only is it impossible to distinguish between $\mathrm{Mu}^{+}$and $\mathrm{H}^{-}$by $\mu^{+} \mathrm{SR}$ $[14,29]$, but the $\mathrm{Mu}^{+}$is lighter in mass than $\mathrm{H}^{-}$, so it expectedly will move faster than the $\mathrm{H}^{-}$which may result in a lower $E_{A, \Gamma}$. Initial $\mu^{-} \mathrm{SR}$ measurements have been performed for $\mathrm{MgH}_{2}$ to decouple $\mathrm{Mu}^{+} / \mathrm{H}^{-}$motion and to understand how they contribute to the overall mobility observed in $\mu^{ \pm} \mathrm{SR}$ experiments $[29,45]$. Therefore, we conclude that above $150 \mathrm{~K}$, $\mathrm{Mu}^{+}$diffusion and $\mathrm{H}^{-}$anion reorganization contribute to the overall mobility in $\mathrm{YH}_{\sim 1.9}$ thin films.

\section{B. Yttrium oxyhydride, $\mathrm{YH}_{3-2 x} \mathrm{O}_{x}$ \\ 1. Transverse field}

Yttrium oxyhydrides require a different analysis of the $\mu^{+} \mathrm{SR}$ data. This is due to the presence of both $\mathrm{H}^{-}$and $\mathrm{O}^{2-}$ in the compound, where both ions can influence the muon behavior. In addition to various $\mu^{+}-\mathrm{H}^{-}$interactions, the muon can bond to $\mathrm{O}^{2-}$ as $\mu^{+}-\mathrm{O}^{2-}$, seen in many oxides $[16,46,47]$. Further, we must consider that the addition of oxygen leads to the appearance of semiconducting properties. Thus, we follow a different type of analysis for $\mathrm{YH}_{3-2 x} \mathrm{O}_{x}$ (compared to $\mathrm{YH}_{2-\delta}$ ), acknowledging these aspects.

Unlike $\mathrm{YH}_{2-\delta}$ [Eq. (1)], the TF data for the $\mathrm{YH}_{3-2 x} \mathrm{O}_{x}$ thin films (see SM [18], Fig. S3) were fitted by an exponentially damped cosine:

$$
A(t)=A_{\mathrm{dia}, \mathrm{TF}} \cos (2 \pi v t+\phi) e^{-\lambda_{\mathrm{TF}} t},
$$

where $A_{\mathrm{dia}, \mathrm{TF}}$ is the diamagnetic asymmetry, $v$ is the Larmor frequency, and $\lambda_{\mathrm{TF}}$ is the exponential relaxation rate. In general, a Lorentzian $B_{\text {local }}$ distribution is used to describe a dilute distribution of magnetic moments $[22,48]$ or rapidly fluctuating magnetic fields, either due to fast muon diffusion or due to fast fluctuating magnetic moments $[32,49]$. In our case, the Lorentzian $B_{\text {local }}$ distribution is likely due to the presence of quickly fluctuating magnetic moments near the muon because the $\mathrm{H}^{-}$content in $\mathrm{YH}_{3-2 x} \mathrm{O}_{x}$ is too high $\left(\mathrm{O}^{2-}: \mathrm{H}^{-} \sim 0.5\right)$ to be considered a dilute magnetic system [4].

The $A_{\text {dia,TF }}$ values of the $\mathrm{YH}_{3-2 x} \mathrm{O}_{x}$ films follow a very different trend compared to $\mathrm{YH}_{2-\delta}$ [Fig. 2(a)]. While the asymmetry in $\mathrm{YH}_{2-\delta}$ is high and temperature-independent, the asymmetries of the $\mathrm{YH}_{3-2 x} \mathrm{O}_{x}$ films are reduced and constantly increasing with temperature. In general, we find values smaller than $A_{\text {dia,max }}$ due to $\mathrm{Mu}^{0}$ formation, which is very common in semiconductors and insulators [23-26]. As stated previously, $\mathrm{Mu}^{0}$ is difficult to measure under our experimental conditions and appears rather as a "missing fraction" of the diamagnetic asymmetry. We observe that for more insulating thin films (higher $p_{\text {dep }}$ ), the amount of $\mathrm{Mu}^{0}$ increases, indicating a relationship between the $\mathrm{O}^{2-}: \mathrm{H}^{-}$ratio and the electronic properties of the material. For example, YHO-07 
TABLE II. Summary of the $E_{g}$ energies (from optical measurements), $\mathrm{Mu}^{0}$ fractions $\left(f_{\mathrm{Mu}^{0}}\right)$, and activation energies $\left(E_{A}\right)$ (from $\mu^{+} \mathrm{SR}$ ) for $\mathrm{YH}_{2-\delta}$ and $\mathrm{YH}_{3-2 x} \mathrm{O}_{x}$. $\mathrm{Mu}^{0}$ fractions are from TF measurements and based on $A_{\text {dia,max }}$. The $E_{A, \Gamma}$ is related to $\mathrm{Mu}^{+} / \mathrm{H}^{-}$diffusion, found only in metallic $\mathrm{YH}_{2-\delta}$. The other activation energies are related to the muon transition state model used to describe the muon behavior in the semiconducting oxyhydrides (see text).

\begin{tabular}{|c|c|c|c|c|c|c|c|}
\hline Name & $p_{\text {dep }}(\mathrm{Pa})$ & $E_{g}(\mathrm{eV})$ & $f_{\mathrm{Mu}^{0}}(10 \mathrm{~K})(\%)$ & $f_{\mathrm{Mu}^{0}}(300 \mathrm{~K})(\%)$ & $E_{A, \Gamma}(\mathrm{meV})$ & $E_{A, \text { dia }}(\mathrm{meV})$ & $E_{A, \lambda}(\mathrm{meV})$ \\
\hline $\mathrm{YH}_{2-\delta}$ & 0.4 & & 0 & 0 & $67 \pm 13$ & & \\
\hline YHO-05 & 0.5 & $2.50 \pm 0.05$ & 28 & 17 & & $29 \pm 2$ & $17 \pm 1$ \\
\hline YHO-07 & 0.7 & $2.58 \pm 0.03$ & 30 & 23 & & $45 \pm 2$ & $16 \pm 1$ \\
\hline
\end{tabular}

(which has the largest band gap of $2.58 \pm 0.04 \mathrm{eV}$ and highest $\mathrm{O}^{2-}$ content $\left.[4,5]\right)$ showed the largest $\mathrm{Mu}^{0}$ fraction (Table II).

Interestingly, the $\mathrm{YH}_{3-2 x} \mathrm{O}_{x}$ asymmetries show a temperature dependence, increasing over a broad temperature range. We conclude that this is due to a gradual recovery of the diamagnetic muon state $\left(\mathrm{Mu}^{+}\right.$or $\left.\mathrm{Mu}^{-}\right)$. Such recovery may occur due to $\mathrm{Mu}^{0}$ ionization or charge carrier capture. First, thermally driven $\mathrm{Mu}^{0}$ ionization typically occurs in a narrow temperature range [24,50-52], which is not the case here. $\mathrm{Next}, \mathrm{Mu}^{0}$ formation and capture of thermally excited charge carriers has also been observed in lowly doped semiconductors where $\mathrm{Mu}^{0}$ forms a defect level close to the valence or conduction band [52,53]. However, although our samples likely have low carrier densities (based on their high resistivity $[6-8,54])$, this process should again occur in a narrow temperature range (related to the defect level) and not gradually between $10-300 \mathrm{~K}$. Instead, we propose that $\mathrm{Mu}^{0}$ is not the lowest energy configuration of the muon, and that it undergoes a site change through a transition state, eventually forming $\mathrm{Mu}^{+}[16,55]$.

Such a transition state model has been proposed for several different oxides $\left(\mathrm{TiO}_{2}\right.$ [56], $\mathrm{ZrO}_{2}$ [57], $\mathrm{Nb}: \mathrm{SnO}_{2}$ [58], and $\mathrm{Lu}_{2} \mathrm{O}_{3}$ [55]), and a schematic representation of this model is shown in Fig. 5. A detailed explanation can be found in Ref. [16]. During thermalization, implanted muons may form $\mathrm{Mu}^{0}$ and come to rest at an interstitial site. However, the presence of this $\mathrm{Mu}^{0}$ may cause a lattice distortion. As the lattice relaxes, $\mathrm{Mu}^{0}$ may move to a bound state with a nearby $\mathrm{O}^{2-}$ anion, via a "transition state". The relaxation of the lattice and coupled movement of $\mathrm{Mu}^{0}$ are related to an activation energy, referred to here as $E_{A \text {,dia }}$.

In the bound state, the electron density of the $\mathrm{Mu}^{0}$ shifts to a neighboring cation to form a polaron at the metal cation. The formation of this polaron is associated with an additional activation energy $\left(E_{A, \lambda}\right)$, sometimes called the muon-electron binding energy $[16,57]$. In general, $E_{A, \lambda}$ is lower than $E_{A \text {,dia }}$ $[16,57]$. In this way, although the muon initially forms in-

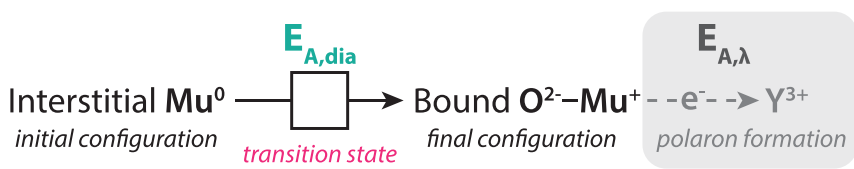

FIG. 5. Schematic image showing the transition state model and the associated activation energies where the muon makes a site change and forms a polaron on the nearest cation. Inspired by Ref. [16]. terstitial $\mathrm{Mu}^{0}$, as the temperature increases, more muons can surmount these energy barriers in favor of the final $\mathrm{Mu}^{+}-\mathrm{O}^{2-}$ state.

The activation energy $E_{A \text {,dia }}$ can be determined based on the fitting of the increasing $\mathrm{Mu}^{+}$fraction [Fig. 6(a)] by a
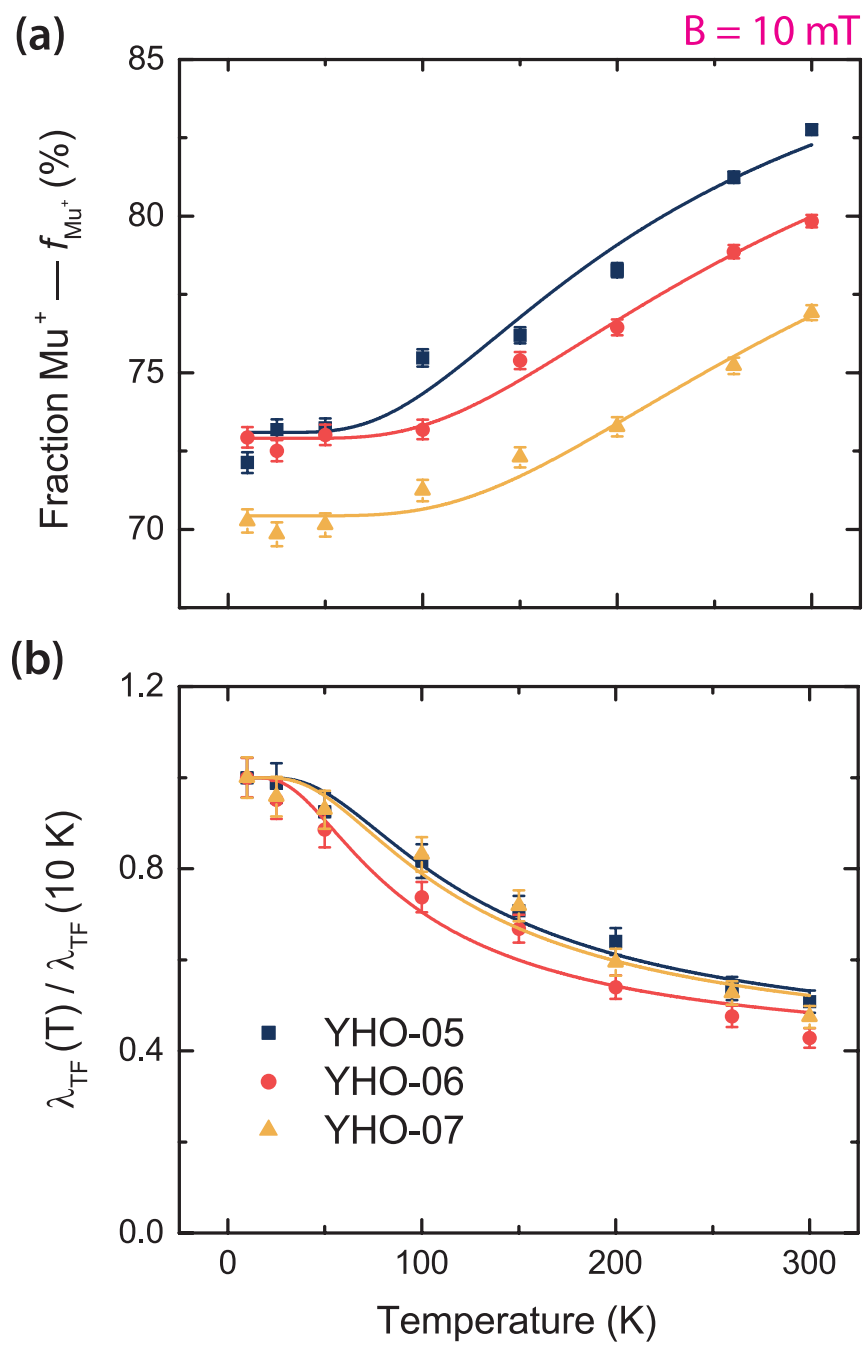

FIG. 6. Boltzmann fitting results of (a) the $\mathrm{Mu}^{+}$fraction and (b) the $\lambda_{\text {TF }}$ for three $\mathrm{YH}_{3-2 x} \mathrm{O}_{x}$ thin films [YHO-05 (navy), YHO-06 (red), YHO-07 (yellow)]. The $\mathrm{Mu}^{+}$fraction is calculated from the TF asymmetry in Fig. 2 by $f_{\mathrm{Mu}^{+}}=100-f_{\mathrm{Mu}^{0}}$. The relaxation rate, $\lambda_{\mathrm{TF}}$, is normalized to the value at $10 \mathrm{~K}$. Equations (9) and (10) are used to obtain the energy barriers (a) $E_{A, \text { dia }}$ and (b) $E_{A, \lambda}$, respectively. 


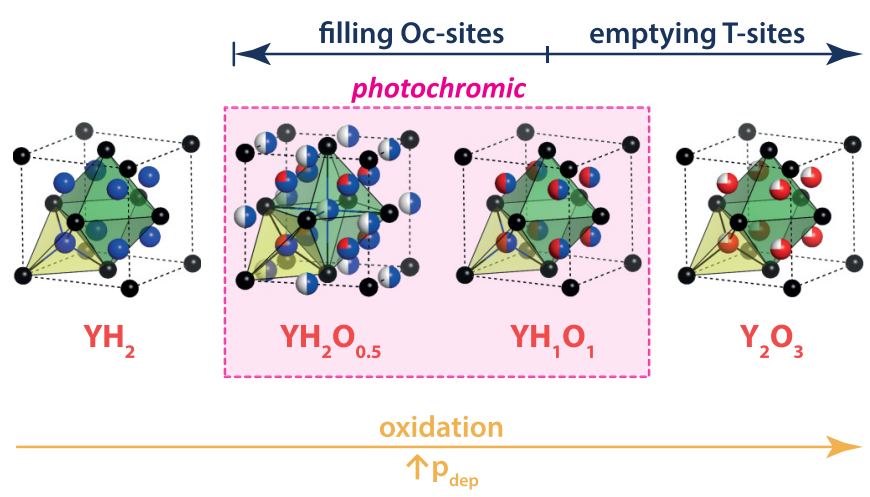

FIG. 7. Schematic representation of yttrium dihydride $\left(\mathrm{YH}_{2}\right)$, two oxyhydrides $\left(\mathrm{YH}_{3-2 x} \mathrm{O}_{x}\right)$, and yttrium oxide $\left(\mathrm{Y}_{2} \mathrm{O}_{3}\right)$, highlighting their structural differences. In this anion-disordered model, partial occupancy of interstitial sites is denoted by multicolored circles: $\mathrm{Y}^{+2 /+3}$ (black), $\mathrm{H}^{-}$(blue), $\mathrm{O}^{2-}$ (red), and vacancies (white). As the $p_{\text {dep }}$ increases, more oxidized compounds are formed, where YHO-05, YHO-06, and YHO-07 likely fall in the photochromic range between $\mathrm{YH}_{2} \mathrm{O}_{0.5}$ and $\mathrm{YHO}$. More detailed explanations can be found in Refs. [4,5,60].

Boltzmann-like equation [59]:

$$
f_{\mathrm{Mu}^{+}}(T)=f_{0}+\frac{\left(100-f_{0}\right) N e^{-E_{A, \mathrm{dia}} / k_{B} T}}{1+N e^{-E_{A, \mathrm{dia}} / k_{B} T}}
$$

where $f_{0}$ is the initial $\mathrm{Mu}^{+}$fraction at $10 \mathrm{~K}, N$ is a density of states parameter (fixed at 1.7), $k_{B}$ is the Boltzmann constant, and $E_{A, \text { dia }}$ is the activation energy for converting interstitial $\mathrm{Mu}^{0}$ to the bound state (Table II).

In general, the values for $E_{A \text {,dia }}$ are lower than what has been reported previously in oxides $(\sim 120-260 \mathrm{meV})$ [16,57]. However, our samples are different from previous work because they are not pure oxides, but contain a significant amount of $\mathrm{H}^{-}$and anion vacancies [4,5]. Values outside this range have also been reported, for example, for CIGS and CZTS thin films which had $E_{A \text {,dia }}$ barriers of $50-70 \mathrm{meV}$ and 100-160 meV, respectively [59]. This could be the result of the nature of the anions (Se or S, respectively), the amount of defects, or other aspects. Differences may also be attributed to structural properties; the $E_{A \text {,dia }}$ values obtained for some monoclinic oxides were consistently larger than those for cubic oxides [57]. Hence, we conclude that $E_{A \text {,dia }}$ depends on the local lattice structure of the material.

Indeed, our values for $E_{A \text {,dia }}$ depend on the $\mathrm{O}^{2-}: \mathrm{H}^{-}$ratio, being higher as the films become more O-rich (higher $p_{\text {dep }}$ ), which is likely due to structural differences between the oxyhydrides. For example, the $\mathrm{O}^{2-}: \mathrm{H}^{-}$ratio determines the quantity and distribution of vacancies in the interstitial sublattice (Fig. 7). Cornelius et al. [4] proposed an anion-disordered model to describe the anion occupancy in $R E \mathrm{H}_{3-2 x} \mathrm{O}_{x}$ thin films, where tetrahedral sites ( $\mathrm{T}$ ) are occupied preferentially by $\mathrm{O}^{2-}$ [60]. Since addition of $\mathrm{O}^{2-}$ leads to removal of two $\mathrm{H}^{-}$, one from an octahedral $(\mathrm{Oc})$ and one from a T site, more O-rich thin films have more Oc (and eventually $\mathrm{T}$ ) vacancies. Interstitial $\mathrm{Mu}^{0}$ likely stops in an Oc vacancy, since this may be the only available site in the films measured here [5]. Thus, the availability of vacancies and their quantity may affect the stability of the interstitial $\mathrm{Mu}^{0}$ state.
In some $R E$ oxides $\left(\mathrm{Y}_{2} \mathrm{O}_{3}[61]\right.$ and $\left.\mathrm{Lu}_{2} \mathrm{O}_{3}[55,62]\right)$, the $\mathrm{Mu}^{0}$ state was described as the lowest energy configuration, opposite from our finding. These oxides are typically characterized by anion vacancies. This suggests that not only the quantity, but the type of available vacancy is important for the muon behavior. Vacant $\mathrm{T}$ and Oc sites differ, with Oc sites being larger and 6-fold coordinated [60]. Thus, the $\mathrm{Mu}^{0}$ state may be stabilized in compounds with many Oc vacancies such as YHO-07, and further stabilized in the presence of additional $\mathrm{T}$ vacancies, as in $\mathrm{Y}_{2} \mathrm{O}_{3}$.

Another reason for the instability of the $\mathrm{Mu}^{0}$ state in oxyhydrides may be the "flexibility" or deformability of their lattices. While our compounds have a face-centered cubic crystal structure, $\mathrm{Y}_{2} \mathrm{O}_{3}$ has a bixbyite structure, which is a distorted fcc lattice [4]. This distorted lattice may be resistant to relaxation around a foreign species like $\mathrm{Mu}^{0}$. Note also that $\mathrm{H}^{-}$is a very polarizable ion [1] which can be mobile in hydrides (see above) and oxyhydrides [6]. Thus, more H-rich oxyhydrides may need less energy for lattice relaxation since this process is related to local atom displacements [16], and $\mathrm{O}^{2-}$ can be considered a hard-shell ion while $\mathrm{H}^{-}$is polarizable.

From the temperature dependence of $\lambda_{\mathrm{TF}}$ [Fig. 2(b)], an activation energy can be determined based on another Boltzmann-like equation [Fig. 6(b)]:

$$
\lambda_{\mathrm{TF}}(T)=\frac{1}{1+N e^{-E_{A, \lambda} / k_{B} T}},
$$

where the empirical parameter $N$ relates to the shape of the binding potential (fixed to 1.7), $k_{B}$ is the Boltzmann constant, and $E_{A, \lambda}$ is the muon-electron binding energy (Table II). The magnitude of $E_{A, \lambda}$ is similar to what has been found for other compounds (5-15 meV) [57] and appears to be independent of the $\mathrm{O}^{2-}: \mathrm{H}^{-}$ratio.

$E_{A, \lambda}$ describes the shift of the $\mathrm{Mu}^{0}$ electron density toward the nearest metal cation, reducing its oxidation state $\left(\mathrm{Y}^{3+} \rightarrow\right.$ $\mathrm{Y}^{2+}$ ) and forming a polaron [16,57]. At low temperatures, the electron is close to the $\mathrm{Mu}^{+}$in the bound state, resulting in a larger relaxation rate. But as the temperature increases, the electron gradually moves closer to the cation, reducing the hyperfine interactions between $\mathrm{Mu}^{+}$and the polaronic electron, and leading to a lower relaxation rate. The $\mathrm{Mu}^{0}$ electron density in $\mathrm{Y}_{2} \mathrm{O}_{3}$, for example, was described as being centered on the cation by DFT [61]. As well, yttrium can be stable in a divalent oxidation state (able to form compounds such as $\mathrm{YH}_{2-\delta}$ ) and has a relatively small reduction potential $\left[E^{0}\left(\mathrm{Y}^{3+} / \mathrm{Y}^{2+}\right)=-2.81 \mathrm{~V}\right]$ [63]. Such cation reduction has also been seen in $\mathrm{ZrO}_{2}$ [57] and $\mathrm{TiO}_{2}$ [56] where the oxidation state of $\mathrm{Ti}$, for example, was reduced from $4+$ to $3+$ upon formation of a polaron and transition to the bound $\mathrm{Mu}^{+}-\mathrm{O}^{2-}$ configuration. In this way, the transition state model explains the temperature-dependent trends of both the $\mathrm{Mu}^{+}$fraction $\left(A_{\mathrm{dia}, \mathrm{TF}}\right)$ and $\lambda_{\mathrm{TF}}$.

\section{Zero field}

As we showed in previous sections on $\mathrm{YH}_{2-\delta}$, $\mathrm{ZF}$ measurements can be used to both probe different muon-sample interactions compared to the TF, and to give further evidence for ideas derived from TF results. Similarly, for the oxyhy- 
drides, we use the $\mathrm{ZF}$ results to comment on the behavior of $\mathrm{H}^{-}$(to which the TF was insensitive), and to support the transition state model we proposed based on the TF temperature trends.

In the $\mathrm{YH}_{2-\delta}$ sample, the $\mathrm{ZF}$ data were considered in terms of $\mathrm{H}-\mu$ complex formation and local mobility of $\mathrm{H}^{-}$ions. A similar $\mathrm{H}-\mu$ complex was found in a metallic oxyhydride $\left(\mathrm{BaTiO}_{3-x} \mathrm{H}_{x}\right)$ [15], showing that such a complex can form despite the presence of $\mathrm{O}^{2-}$ and regardless of the (large) $\mathrm{O}^{2-}: \mathrm{H}^{-}$ratio. However, in our $\mathrm{YH}_{3-2 x} \mathrm{O}_{x}$ films, this is not the case. The muon behavior more closely resembles the transition state model and the $\mathrm{H}-\mu$ equation did not fit the data well (see SM [18], Fig. S3), possibly due to the amount of defects and anion disorder present in our samples, as well as $\mathrm{Mu}^{0}$ formation. Though a small fraction of muons may have formed this complex, other interactions between the muon and sample environment are more relevant. Thus, a different treatment of the data follows for the $\mathrm{YH}_{3-2 x} \mathrm{O}_{x}$ films.

The $\mathrm{ZF}$ data for the $\mathrm{YH}_{3-2 x} \mathrm{O}_{x}$ films was fitted by a phenomenological description of the static Gaussian KuboToyabe (KT) equation:

$$
A(t)=A_{\mathrm{KT}}\left(\frac{1}{3}+\frac{2}{3}\left[1-\left(\Delta_{\mathrm{ZF}} t\right)^{2}\right] e^{-\frac{1}{2}\left(\Delta_{\mathrm{ZF}} t\right)^{2}}\right) e^{-\lambda_{\mathrm{ZF}} t}+A_{\mathrm{NR}},
$$

where $A_{\mathrm{KT}}$ is the asymmetry related to the Kubo-Toyabe function, $\Delta_{\mathrm{ZF}}$ is the Gaussian relaxation rate, $\lambda_{\mathrm{ZF}}$ is the exponential relaxation rate, and $A_{\mathrm{NR}}$ is related to the fraction of nonrelaxing muons. Without the $\exp \left(-\lambda_{\mathrm{ZF}} t\right)$ term, the static Gaussian KT function describes a muon surrounded by static nuclear spins which generate an isotropic $B_{\text {local }}$ distribution with a Gaussian shape [22]. However, if there are also rapidly fluctuating interactions of the muon with surrounding electrons, adding the exponential term significantly improves the fits by including the different contributions to the muon spin depolarization $[13,45,48]$. This is especially important when dealing with the combined contributions of nuclei and electrons which normally result in relaxation rates of very different magnitudes $[13,32,45]$. We note that longitudinal field (LF) measurements are also useful for discriminating between different phenomena, and are open for future study.

Figure 8(a) shows the asymmetry contributions from Eq. (11) compared to the TF asymmetry from Eq. (8). The sum of the $\mathrm{ZF}$ asymmetry components $\left(A_{\mathrm{KT}}+A_{\mathrm{NR}}\right)$ is equivalent to $A_{\mathrm{TF}}$. Further, the phenomenological KT equation represents the majority of the data, with a small contribution from the nonrelaxing muon fraction. Overall, the asymmetry values support the use of the chosen fitting equation. However, $A_{\mathrm{KT}}$ accounts for the asymmetry of both Gaussian and exponential contributions simultaneously and cannot be used to distinguish them.

More detailed information about the muon environment can be obtained from the relaxation rates shown in Fig. 8(b). The Gaussian relaxation rate can be related to nearby nuclei (mainly $\mathrm{H}^{-}$). The magnitude of $\Delta_{\mathrm{ZF}}\left(\sim 0.2 \mu \mathrm{s}^{-1}\right)$ is almost equivalent to the $\sigma_{\mathrm{ZF}}$ found in $\mathrm{YH}_{2-\delta}$ [Fig. 4(c)] for static $\mathrm{H}^{-}$, in line with the fact that the films we measured are oxyhydrides (or oxygenated $\mathrm{YH}_{x}$ ) with a significant quantity of $\mathrm{H}^{-}$rather than the oxide $\left(\mathrm{Y}_{2} \mathrm{O}_{3}\right)$. Further, the largely temperature-independent trend of $\Delta_{\mathrm{ZF}}$ suggests that $\mathrm{H}^{-}$is

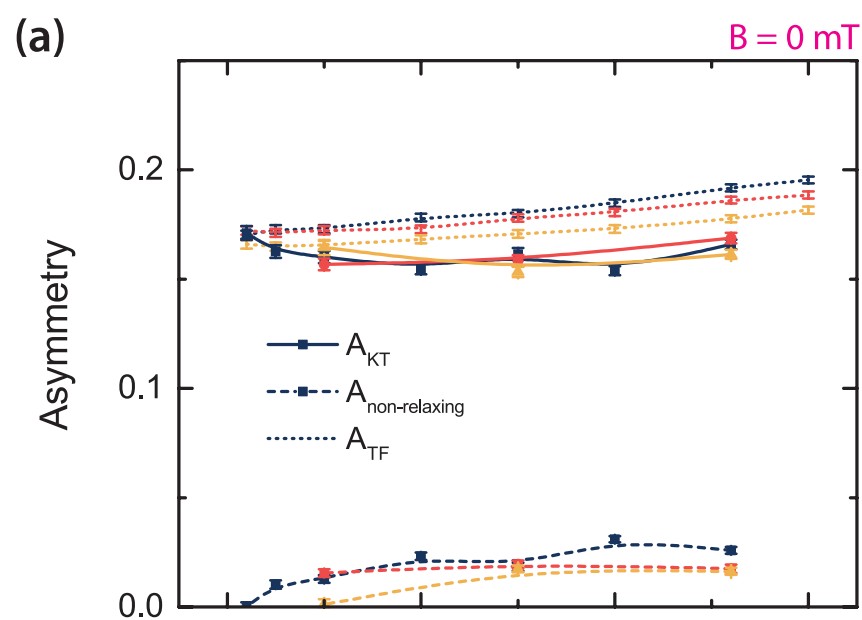

(b)

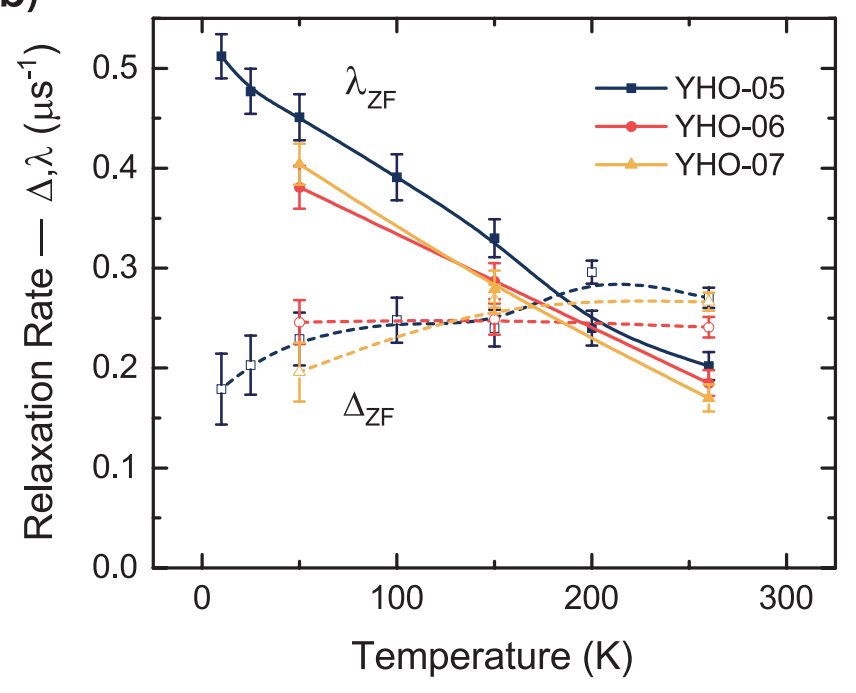

FIG. 8. A summary of the fitting parameters obtained from the analysis of the $\mathrm{ZF} \mathrm{YH}_{3-2 x} \mathrm{O}_{x}$ data with Eq. (11). (a) The asymmetry contributions from the $\mathrm{ZF}\left(A_{\mathrm{KT}}+A_{\mathrm{NR}}\right)$ are compared to the $\mathrm{TF}$ values. The sum of the two $\mathrm{ZF}$ components is equivalent to the TF value. (b) The two relaxation rates contributing to the total muon depolarization, where $\lambda_{\mathrm{ZF}}$ is related to quickly fluctuating electron moments, while $\Delta_{\mathrm{ZF}}$ is related to nuclear moments, mostly $\mathrm{H}^{-}$. Lines are guides to the eye.

static within the muon lifetime $(2.2 \mu \mathrm{s})$ for all three measured oxyhydrides. It is possible that $\mathrm{H}^{-}$mobility in these compounds is hindered by the presence of $\mathrm{O}^{2-}$ ions, anion disorder, and other effects. Thus, the onset temperature for diffusion may be above $300 \mathrm{~K}$. In general, $\mathrm{H}^{-}$conductivity in oxyhydrides has been reported for temperatures in the range of $400-600 \mathrm{~K}[6,64]$. Further, some rare-earth metal oxyhydrides are predicted to form anion-disordered compounds, reducing the ionic mobility [7].

The exponential relaxation rate, on the other hand, can be related to electron moments and serves as further evidence in favor of the transition state model. We showed that in $\mathrm{YH}_{2-\delta}$, relaxation rates related to nuclei with large magnetic moments (specifically $\mathrm{H}^{-}$) are in the range of $\sim 0.22 \mu \mathrm{s}^{-1}$. Thus, the magnitude of $\lambda_{\mathrm{ZF}}\left(\sim 0.5 \mu \mathrm{s}^{-1}\right)$ cannot be explained by considering nuclei in proximity to $\mathrm{Mu}^{+}$. Rather, $\lambda_{\mathrm{ZF}}$ has 
to be the result of electrons near $\mathrm{Mu}^{+}$, since only such interactions between $\mathrm{Mu}^{+}$and electronic moments can lead to larger depolarization rates compared to interaction with nuclear moments $[13,32,45]$. This is relevant to the transition state model where in the final configuration, $\mathrm{Mu}^{+}$is sensitive to the magnetic moment of the neighboring polaronic electron and the $\mathrm{Mu}^{+}$-polaron distance increases with temperature, leading to a gradual reduction of the exponential relaxation rate, $\lambda_{\mathrm{ZF}}$.

\section{In situ illumination}

Rare-earth metal oxyhydride thin films exhibit a photochromic effect [8], the origin of which is not yet clear. We performed in situ $\mu^{+} \mathrm{SR}$ experiments to see if the muon behavior is affected by optical illumination. This muon behavior is discussed in terms of the transition state model proposed in Sec. III B 1, where samples measured "in the dark" exhibit a gradual, thermally driven $\mathrm{Mu}^{+}$recovery.

Oxyhydride samples were illuminated and their photochromic response is shown in Fig. 9(a). A transmission spectrum is collected every $15 \mathrm{~s}$ from which the average transmittance $(\lambda=450-1000 \mathrm{~nm})$ is calculated with respect to time. The relative contrast is plotted to normalize for the initial transmittance of the samples which differ slightly. The samples were illuminated for $2 \mathrm{~h}$ by a $385 \mathrm{~nm}$ LED (gray area in figure), during which the samples became opaque. After illumination, the original transparent state was recovered within $2 \mathrm{~h}$. The amount of color change which occurred is called the contrast and the time with which the samples return to their transparent state is called the bleaching time, $\tau_{B}$ (Table I). The calculations for these quantities are discussed in Refs. [2,3,5]. Higher $p_{\text {dep }}$ samples had the lowest photochromic contrast but the fastest $\tau_{B}$, observed also in our detailed studies of the $p_{\text {dep }}$ dependence of the photochromic effect in various $R E \mathrm{H}_{3-2 x} \mathrm{O}_{x}$ [5].

Twin samples (deposited alongside those described above) were measured by $\mu^{+} \mathrm{SR}$ using in situ illumination from a $365 \mathrm{~nm}$ LED. Only TF measurements were performed and were fitted by Eq. (8), where two parameters are considered for this discussion: $\lambda_{\mathrm{TF}}$ and $A_{\mathrm{TF}, \text { dia. }}$. While $\lambda_{\mathrm{TF}}$ was constant during the measurement (see SM [18], Fig. S4), $A_{\mathrm{TF}, \mathrm{dia}}$ showed a clear difference upon illumination [Fig. 9(b)]. As well, it shows a $p_{\text {dep }}$ dependence, with the largest change in the asymmetry for YHO-05, which also had the greatest photochromic contrast.

After many hours of bleaching (and a heating program described in the Supplemental Material), the samples returned to their initial value of $A_{\mathrm{TF}, \text { dia }}$ (Fig. 9(b) and see SM [18], Fig. S5). The difference in kinetics is likely due to the lower experimental temperature, which generally results in slower bleaching rates $[10,65,66]$. Nevertheless, these observations clearly establish a relation between the optical and muon behavior under illumination, showing both reversible and $p_{\text {dep-dependent changes. }}$

An increased asymmetry during illumination suggests a larger diamagnetic $\left(\mathrm{Mu}^{+}\right.$or $\left.\mathrm{Mu}^{-}\right)$and a smaller paramagnetic $\left(\mathrm{Mu}^{0}\right)$ fraction. This can happen for several reasons [Fig. 10(a)]. In general, we consider three muon populations in the oxyhydrides [16]: (1) diamagnetic $\mathrm{Mu}^{+}$which form the
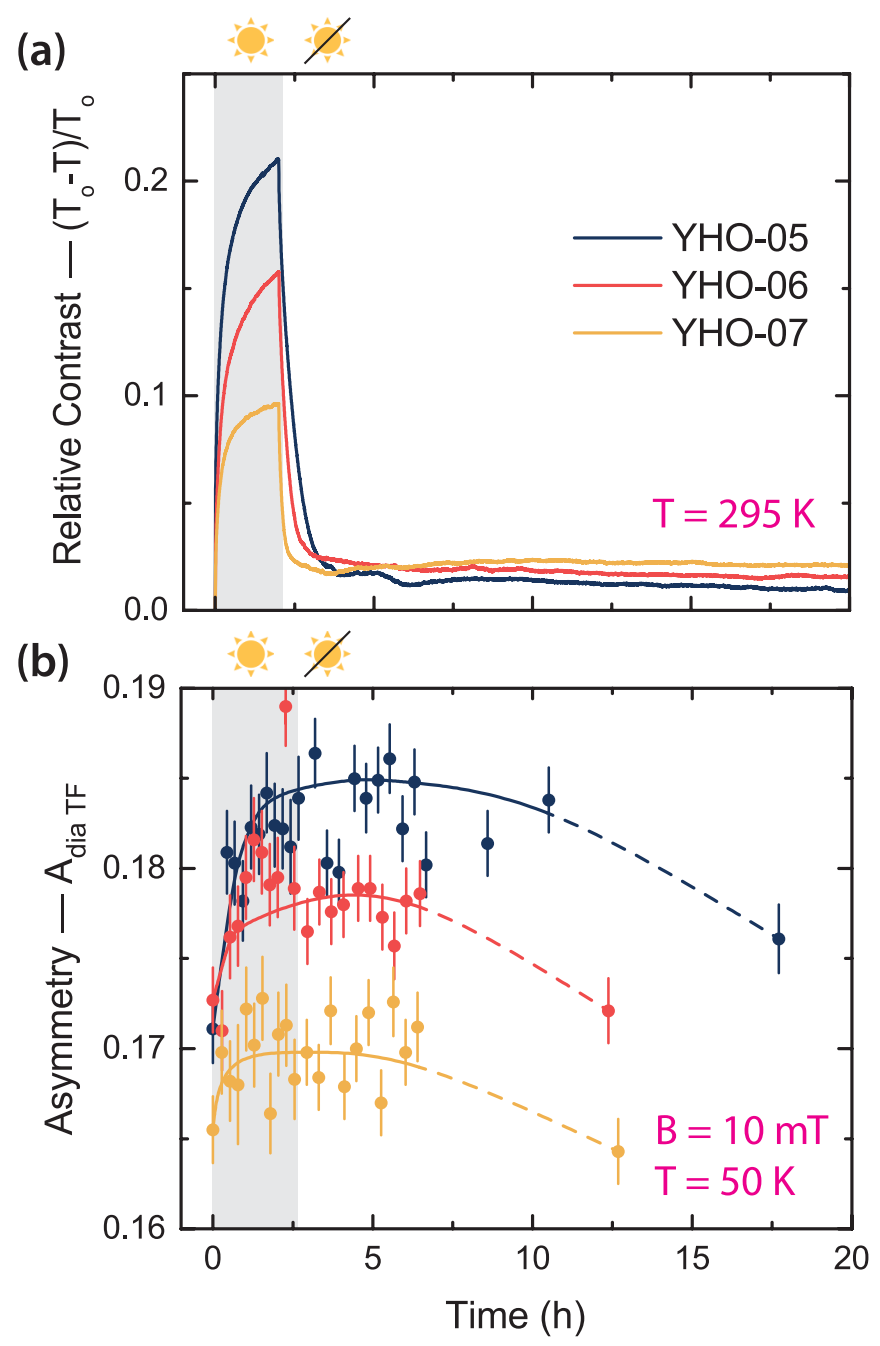

FIG. 9. (a) The relative optical contrast with time for the $\mathrm{YH}_{3-2 x} \mathrm{O}_{x}$ films under illumination of a $385 \mathrm{~nm}$ LED for $2 \mathrm{~h}$ at room temperature. The gray area indicates when the LED was on and inducing photodarkening. (b) TF asymmetry $\left(A_{\mathrm{TF}, \text { dia }}\right)$ from in situ illumination by a $365 \mathrm{~nm}$ LED for $\sim 2 \mathrm{~h}$ at $50 \mathrm{~K}$. Between the last two points in every line, the temperature was increased incrementally to $300 \mathrm{~K}$ and then back down to $50 \mathrm{~K}$ to promote complete thermal bleaching (see SM [18], Fig. S5).

bound state promptly, (2) muons which start as $\mathrm{Mu}^{0}$, but form the bound state $\left(\mathrm{Mu}^{+}\right)$via a transition state (delayed by lattice relaxation), and (3) interstitial $\mathrm{Mu}^{0}$ which account for the "missing fraction" of the asymmetry. The latter two may be converted to a diamagnetic state by photoinduced ionization $[67,68]$. The incident light energy was larger than the band gap, $E_{g}$, thus, sufficient to ionize in-gap states created by $\mathrm{Mu}^{0}$ [61]. However, this is an unlikely mechanism because we expect the recovery of the $\mathrm{Mu}^{0}$ state after light is ceased to be faster than what is shown in our results. Here the asymmetry remained elevated for several hours. So, this process cannot be treated as the main reason for the $A_{\mathrm{TF}, \mathrm{dia}}$ increase upon illumination.

Generally, we assume that light exposure results in charge carrier generation. Since the energy of the photodarkening LED must be larger than $E_{g}$ to induce photochromism, the first 
(a)

(b)
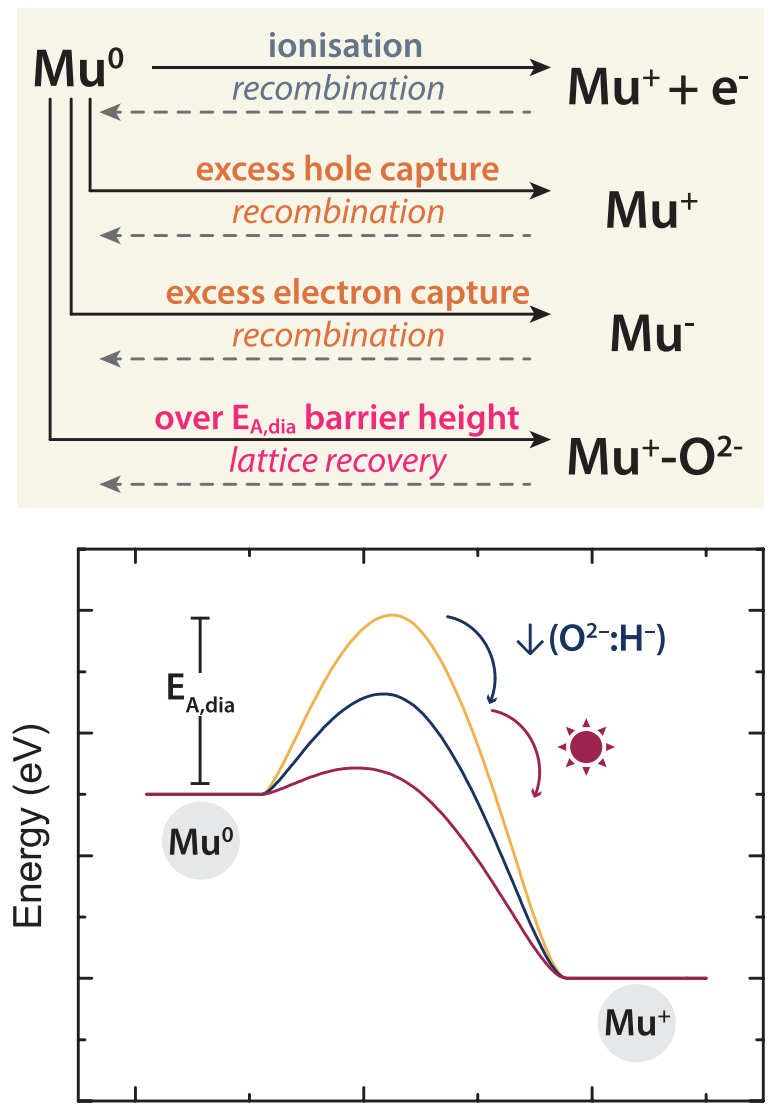

Reaction Coordinate

FIG. 10. (a) Reactions showing the possible routes $\mathrm{Mu}^{0}$ may take to form a diamagnetic species $\left(\mathrm{Mu}^{+}\right.$or $\left.\mathrm{Mu}^{-}\right)$under illumination. Reverse arrows indicate the return to the original $\mathrm{Mu}^{0}$ state once illumination is stopped. (b) Schematic representation of $E_{A \text {,dia }}$, an energy barrier involved in the transition state model. $E_{A \text {,dia }}$ decreased for more $\mathrm{H}$-rich samples, and further decreased by in situ illumination. $\lambda_{\mathrm{TF}}$, and thus $E_{A, \lambda}$, was unaffected by illumination (see SM [18], Fig. S4). Image inspired by Ref. [16].

step in the mechanism is the formation of electron-hole pairs. Carrier generation can lead to a decrease of the initial $\mathrm{Mu}^{0}$ population after thermalization and a lower $\mathrm{Mu}^{0}$ formation probability due to electron shielding. However, this would typically require a very high carrier density $\left(>10^{18} \mathrm{~cm}^{-3}\right)$ $[24,69]$ which is likely not attained here under illumination [6-8,54].

Photogenerated carriers can also be captured by $\mathrm{Mu}^{0}$ to form diamagnetic $\mathrm{Mu}^{+}$or $\mathrm{Mu}^{-}$[Fig. 10(a)]. However, there must again be a sufficient number of charge carriers available $\left(>10^{17} \mathrm{~cm}^{-3}\right)[24,69]$ such that $\mathrm{Mu}^{0}$ formation and charge carrier capture occur very rapidly (within nanoseconds) and result in a larger diamagnetic fraction. Further, the relaxation of $A_{\mathrm{TF} \text {,dia }}$ back to its original value is slower than expected for charge carrier recombination [70], suggesting that this is not the reason for the observed reversible increase in $A_{\mathrm{TF} \text {,dia }}$.

In fact, the photochromic mechanism likely consists of many more reaction steps following photogeneration of carriers. For example, in $\mathrm{Cu}$-doped $\mathrm{Ag}$-halide glasses, electron-hole pair generation is followed by trapping, with $h^{+}$oxidizing copper $\left(\mathrm{Cu}^{+} \rightarrow \mathrm{Cu}^{2+}\right)$ and $e^{-}$reducing silver $\left(\mathrm{Ag}^{+} \rightarrow \mathrm{Ag}^{0}\right)$, eventually forming light-absorbing $\mathrm{Ag}^{0}$ clusters $[9,10]$. Trapping of the carriers leads to a prolonged darkened state and a bleaching process which is not instantaneous. Therefore, the aforementioned processes [shown in Fig. 10(a)] are not the main contributors to the increased $A_{\mathrm{TF}, \mathrm{dia}}$. Rather, the photogenerated carriers may be trapped at a defect, ion, or atom and the formation of a light-absorbing species affects the muon behavior.

Similarly to the case of $\mathrm{Cu}$-doped Ag-halide glasses, a number of papers attribute the photochromic effect in $R E \mathrm{H}_{3-2 x} \mathrm{O}_{x}$ thin films to the formation of metallic clusters (discussed more in Sec. IV A) due to the increased electronic conductivity and decreased optical transparency of the darkened state [8]. If metallic $\left(n>10^{21} \mathrm{~cm}^{-3}\right)$ clusters are formed and are large enough $(\geqslant 1 \mathrm{~nm})$ to create sufficient static shielding of the implanted muon $\left(\mathrm{Mu}^{+}\right)$, this can prevent some $\mathrm{Mu}^{0}$ formation, resulting in a higher diamagnetic fraction upon illumination. The increases we see in $A_{\mathrm{TF}, \mathrm{dia}}$ [Fig. 9(b)] from the transparent $(t=0 \mathrm{~h})$ to the photodarkened state $(t \sim 2 \mathrm{~h})$ (YHO-05: 8\%, YHO-06: 5\%, YHO-07: 3\%) would imply that the volume fraction of a metallic phase decreases with increasing $\mathrm{O}^{2-}$ content, consistent with the observed decrease in photochromic contrast [Fig. 9(a)].

However, a volume fraction of $\sim 6 \%$ of formed metallic clusters (from Ref. [71]) is hard to reconcile with the large reduction in the $\mathrm{Mu}^{0}$ fraction observed between the transparent and photodarkened states. This volume fraction would only account for $\sim 30 \%-40 \%$ of the observed reduction in $\mathrm{Mu}^{0}$ fraction. Further, the temperature-dependent recovery of $A_{\text {TF,dia }}$ (see SM [18], Fig. S5) is difficult to explain simply by metallic phase formation as we expect similar recovery temperatures for all three films, which did not happen. The most notable difference is between YHO-05 and YHO-07 which return to their original $A_{\mathrm{TF}, \mathrm{dia}}$ at $\sim 260 \mathrm{~K}$ and $\sim 100 \mathrm{~K}$, respectively [see SM [18], Figs. S5(a)-S5(c)], showing that the light-induced changes in asymmetry are not a pure volume fraction effect. Instead, it appears that each $\mathrm{YH}_{3-2 x} \mathrm{O}_{x}$ follows a different energy barrier height for bleaching to the transparent state.

Thus, although metallic cluster formation may be partially responsible for the increased $A_{\mathrm{TF}, \mathrm{dia}}$, we propose that the main phenomenon driving this reversible behavior is related to the transition state model (Sec. III B 1). Within this model, $A_{\mathrm{TF}, \mathrm{dia}}$ depends on the energy barrier $E_{A \text {,dia }}$, which is associated with the local lattice structure and the activation energy of lattice relaxation. An increase in $A_{\mathrm{TF} \text {,dia }}$ suggests a decrease in $E_{A, \text { dia }}$ during illumination, leading to a higher formation probability of the bound $\mathrm{Mu}^{+}-\mathrm{O}^{2-}$ state as more muons can surmount this lower energy barrier [Fig. 10(b)]. This can happen as a result of a local lattice change which facilitates the $\mathrm{Mu}^{0}$ site change or causes the interstitial $\mathrm{Mu}^{0}$ state to become less stable. Interestingly, the reversible increase in $A_{\mathrm{TF}, \mathrm{dia}}$ shows the same trend as a function of $p_{\text {dep }}$ as the photochromic contrast and $E_{A \text {,dia }}$, supporting the connection between the transition state model, photochromism, and the importance of structural properties in the photochromic effect.

Along these lines, we conclude that a reversible change in the interstitial sublattice occurs during illumination, after which, some light-absorbing species is formed (e.g., metal- 
lic clusters). The lattice change depends on factors such as the $\mathrm{O}^{2-}: \mathrm{H}^{-}$ratio, amount and distribution of anion vacancies, ease of lattice distortion, and other structural properties. Eventually, oxyhydrides with lower $\mathrm{H}^{-}$content (and lower photochromic contrast) may have smaller volume fractions of these metallic phases. Although this conclusion is possible, other theories are discussed below to suggest alternative steps after the light-induced local lattice changes (e.g., local lattice relaxations, octahedral site occupancy changes).

\section{DISCUSSION: IMPLICATIONS FOR THE PHOTOCHROMIC EFFECT}

When exposed to UV light, photochromic $\mathrm{YH}_{3-2 x} \mathrm{O}_{x}$ thin films exhibit a reversible and $p_{\text {dep }}$-dependent optical response. Remarkably, we observe a correlated (reversible) increase in the diamagnetic asymmetry of the muon during illumination. This suggests that the energy barrier for lattice relaxation, $E_{A \text {,dia }}$, is lowered by exposure to UV light, shown schematically in Fig. 10(b). To understand this change in $E_{A \text {,dia }}$ in terms of the structural properties of $\mathrm{YH}_{3-2 x} \mathrm{O}_{x}$, we will discuss the various mechanisms proposed for the photochromic effect.

The first step of the photochromic mechanism in $R E \mathrm{H}_{3-2 x} \mathrm{O}_{x}$ is the formation of electron-hole pairs since the energy of the incident light must be larger than the $E_{g}$ to induce photodarkening. The subsequent steps and the identity of the light-absorbing species are debated. Several ideas have been proposed and are summarized below, discussed in the context of our $\mu^{+} \mathrm{SR}$ results.

\section{A. Formation of metallic centers by $\mathrm{O}^{2-}$ or $\mathrm{H}^{-}$diffusion}

In analogy to $\mathrm{Cu}$-doped $\mathrm{Ag}$-halide glasses [9,10,12,65], several reports have suggested that the photochromic effect in $R E \mathrm{H}_{3-2 x} \mathrm{O}_{x}$ is due to metallic cluster formation. Ellipsometry studies on $\mathrm{YH}_{3-2 x} \mathrm{O}_{x}$ thin films, for example, suggested the formation of metallic clusters (volume fraction $\sim 6 \%$ ) in a semiconducting matrix, stating that even $2 \%$ of such clusters would result in a decrease in transparency of $~ 30 \%$ [71,72]. We explained also in Sec. III C that metallic cluster formation (which follows from changes in the interstitial sublattice) may explain up to $\sim 30 \%$ of our observed changes in $A_{\mathrm{TF} \text {,dia }}$.

However, Baba et al. [73] proposed that such metallic phases are formed due to $\mathrm{O}^{2-}$ diffusion, causing a reversible lattice contraction and formation of a highly oxidized thinfilm surface. Such $\mathrm{O}^{2-}$ diffusion is hard to reconcile with the results of our study. We observed photochromism at temperatures as low as $50 \mathrm{~K}$, where $\mathrm{O}^{2-}$ ions are usually immobile, even in $\mathrm{O}^{2-}$ conducting oxides [74]. Also, mobility in oxyhydrides is generally seen to involve only $\mathrm{H}^{-}$, with $\mathrm{O}^{2-}$ being regarded as stationary $[6,7,64,75,76]$. Likewise, we find that $\mathrm{O}^{2-}$ behaves as a hard-shell atom, compared to the polarizable $\mathrm{H}^{-}$ion, making $\mathrm{O}^{2-}$ diffusion difficult.

Alternatively, metallic centers can be formed by $\mathrm{H}^{-}$, rather than $\mathrm{O}^{2-}$, mobility. $\mathrm{H}^{-}$has been reported as mobile in metals [77], hydrides (see above), and oxyhydrides [6,64]. As well, an NMR study showed that a fraction of mobile $\mathrm{H}^{-}$disappear or become stationary upon illumination [11].

We note that, prior to illumination, we did not observe $\mathrm{H}^{-}$mobility in the oxyhydrides [Fig. 2(b), trend of $\lambda_{\mathrm{TF}}(T)$ ], contrary to this room temperature NMR study [11]. However, this apparent discrepancy may be due to the timescale of a $\mu^{+}$SR experiment, since mobility should occur within the muon lifetime $(2.2 \mu \mathrm{s})$, while NMR is sensitive to slower dynamics. So, $\mathrm{H}^{-}$mobility in these materials may be slow with respect to the muon lifetime, making $\mu^{+}$SR insensitive to this under our experimental conditions. Slow $\mathrm{H}^{-}$dynamics could potentially explain the prolonged darkened state and bleaching kinetics $[2,3,5]$, where $\mathrm{H}^{-}$diffusion may act as the rate-limiting step for the formation/dissolution of metallic domains.

Alternatively, a DFT study predicted a mobility bottleneck for $R E \mathrm{H}_{3-2 x} \mathrm{O}_{x}$ containing certain cations [7], with our photochromic materials falling in the ion-insulating regime $[2,3,5]$. This implies that long-range $\mathrm{H}^{-}$diffusion could be hampered in our photochromic materials, and may be unrelated to the underlying mechanism. Short-range mobility, though, may still play a role.

\section{B. Hydroxide formation}

Another possibility is the formation of hydroxides $\left(\mathrm{H}^{+}-\mathrm{O}^{2-}\right)$ upon illumination, as observed in mayenite $\left(12 \mathrm{CaO}_{3} \cdot 7 \mathrm{Al}_{2} \mathrm{O}_{3}\right)$ [78-81]. In this oxide, UV light exposure causes a reaction where $\mathrm{H}^{-}$ions are converted into hydroxides and release electrons, improving the conductivity and the optical absorption of the originally insulating oxide. This reaction is reversible and influenced by temperature.

As it appears to match some of our observations, the formation of a hydroxide species in our materials seems worthy of future investigation (e.g., IR spectroscopy). The proposed hydroxide may be analogous to the bound $\mathrm{Mu}^{+}-\mathrm{O}^{2-}$ configuration, where $\mathrm{Mu}^{+}$mimics $\mathrm{H}^{+}$. This suggests that hydroxide formation may be possible in our materials, assuming the muon behaves similarly to hydrogen which is not always the case [57].

However, a recent DFT study reported that, at room temperature, the hydroxide is unstable in $\mathrm{YH}_{x} \mathrm{O}_{y}$ and tends to dissociate quickly, prompting the authors to exclude this mechanism [82]. More detailed studies of this reaction could be useful for determining the stability of the hydroxide species and its role in photochromism. In particular, the initial charge state of hydrogen can be important since, in our transition state model, $\mathrm{Mu}^{0}$ is an analog of atomic hydrogen, $\mathrm{H}^{0}$, not $\mathrm{H}^{-}$. It is possible that during illumination $\mathrm{H}^{0}$ is formed at the $\mathrm{Oc}$ (octahedral) sites of $\mathrm{YH}_{3-2 x} \mathrm{O}_{x}$ and acts as a mobile fraction, able to form a hydroxide. Such details may be investigated by NMR.

\section{Color centers}

The most important structural characteristics of our oxyhydrides are the vacancy concentration, vacancy type, and possibly the deformability of the lattice. In general, we expect that the presence of fewer Oc vacancies (as occurs in more $\mathrm{H}$-rich $\mathrm{YH}_{3-2 x} \mathrm{O}_{x}$ ) and a more deformable lattice lead to the lowering of $E_{A, \text { dia }}$ by either lowering the stability of the interstitial $\mathrm{Mu}^{0}$ state or allowing for easier lattice relaxation. Since this energy barrier decreases further during illumination, some of these structural properties changed, such as the number 
of available Oc vacancies. This raises the question, what is occupying these vacancies during illumination?

One explanation is that color centers have formed. These were reported in other photochromic materials $[9,83]$ and rare-earth compounds are known to form color centers in some situations [66,84]. The most basic types are known as F centers, where one electron is captured in a cation vacancy $\left(\mathrm{F}^{+}\right)$or two electrons are trapped in an anion vacancy $\left(\mathrm{F}^{-}\right)$ $[9,84]$.

The presence of a paramagnetic electron $\left(\mathrm{F}^{+}\right)$could lead to very fast depolarization of a nearby muon due to its hyperfine coupling with the muon. This would result in a higher $\lambda_{\text {dia,TF }}$ upon illumination, contrary to our observations. Therefore, it is unlikely that an $\mathrm{F}^{+}$-type center is responsible for the photochromic effect here.

However, the capture of two electrons by an Oc vacancy $\left(\mathrm{F}^{-}\right)$might not impact the muon depolarization rate because these electrons would be paired. Also, there would be fewer Oc vacancies available, which seems to promote the $\mathrm{Mu}^{+}-\mathrm{O}^{2-}$ configuration by lowering $E_{A \text {,dia }}$ and increasing $A_{\mathrm{dia}, \mathrm{TF}}$. Although this appears to agree with our in situ $\mu^{+}$SR results, a $\mathrm{Y}^{3+}\left(\mathrm{F}^{-}\right)$center absorbs specific wavelengths [84], and may not necessarily lead to the broad absorption range seen in $R E \mathrm{H}_{3-2 x} \mathrm{O}_{x}$.

It should be noted that many other types of color centers are possible, and future electron paramagnetic resonance measurements may help to elucidate this.

\section{Dihydrogen formation}

A recent computational paper suggested that the photochromic mechanism involves formation and dissolution of a "dihydrogen species," similar to a hydrogen molecule, $\mathrm{H}_{2}$ [82]. This molecule forms between two adjacent $\mathrm{H}^{-}$ions in an octahedral vacancy and creates a shallow donor level close to the conduction band edge. The activation energy for formation, and especially for dissolution, appears to be related to the $\mathrm{O}^{2-}: \mathrm{H}^{-}$ratio. The authors [82] show that dissociation of the molecule has the lowest energy barrier in O-rich compounds, which may explain the faster bleaching kinetics which they also observe. This theory is interesting because it does not require long-range structural rearrangements, with "dihydrogen" formation on a very local scale.

The formation of a "dihydrogen species" in the Oc sites of the lattice could potentially impact the muon behavior. The stability of interstitial $\mathrm{Mu}^{0}$ in an Oc site could change due to the formation of a new molecule in the same octahedron, although the concentration of this dihydrogen species needs to be very large to have a noticeable optical effect. We note that the formation of a hydrogen muonium molecule (as an analog of $\mathrm{H}_{2}$ ) by binding of the interstitial $\mathrm{Mu}^{0}$ to hydrogen is not expected, in view of its large thermodynamic instability compared to the formation of a $\mathrm{H}_{2}$ molecule [85].
However, it is important to note that Chai et al. [82] consider a defect concentration of $\mathrm{O}^{2-}$ in what is essentially a $\mathrm{YH}_{3}$ lattice. In their DFT calculations, the highest $\mathrm{O}: \mathrm{Y}$ ratio is 0.25 . This is significantly lower than the lower boundary we expect for our photochromic thin films $(\mathrm{O}: \mathrm{Y}=0.5-1.0)$ $[4,5,60]$. We believe that the formation of $\mathrm{H}_{2}$ upon illumination requires a more in-depth experimental analysis (e.g., Raman spectroscopy).

\section{CONCLUSION}

Photochromic rare-earth metal oxyhydrides darken reversibly over a broad range of wavelengths when exposed to UV light. Based on $\mu^{+}$SR data, and the electronic and structural properties of our materials, we proposed models to describe the muon behavior in $\mathrm{YH}_{3-2 x} \mathrm{O}_{x}$ thin films with different $\mathrm{O}^{2-}: \mathrm{H}^{-}$ratio, compared to metallic $\mathrm{YH}_{2-\delta}$, which serves as a reference compound.

Briefly, $\mathrm{YH}_{2-\delta}$ shows a $\mathrm{H}-\mu$ complex, which competes with the onset of $\mathrm{Mu}^{+} / \mathrm{H}^{-}$mobility above $150 \mathrm{~K}$. The oxyhydrides, though, are better described by a transition state model, where a transition between interstitial $\mathrm{Mu}^{0}$ and bound $\mathrm{Mu}^{+}-\mathrm{O}^{2-}$ occurs, dependent on the activation energy for lattice relaxation, $E_{A \text {,dia }}$. This barrier decreases with reduced $\mathrm{O}^{2-}: \mathrm{H}^{-}$ratio, and is influenced by structural factors such as the concentration and types of available anion vacancies, and the deformability of the lattice. We also observed polaron formation at the $\mathrm{Y}$ cation together with the formation of the bound state.

Upon illumination of the $\mathrm{YH}_{3-2 x} \mathrm{O}_{x}$ thin films, we observe reversible changes in the diamagnetic asymmetry of the muon related to the transition state model and correlated to the photodarkening. Assuming the muon mimics hydrogen, one option to explain these changes is the formation of a hydroxide during photodarkening, possibly from $\mathrm{H}^{0}$, although a previous DFT study suggested that the hydroxide is unstable at room temperature. Another option is that changes occur in the interstitial sublattice during illumination which lower the energy barrier for muon site changes, thereby increasing the diamagnetic muon fraction. Particularly, there may be fewer available octahedral vacancies in the darkened state which could be populated by electrons (color centers), a dihydrogen species, or $\mathrm{H}^{-}$ions slowly moving between sites. Additional measurements are needed to understand the full reaction scheme for the photochromic effect in these materials.

\section{ACKNOWLEDGMENTS}

The authors would like to acknowledge S. Cornelius for insightful conversations and the staff at the Laboratory for Muon Spin Spectroscopy at PSI for their assistance before, during, and after experiments. This work is supported by the Mat4Sus research program with Project No. 680.M4SF.034, funded by the Dutch Research Council (NWO). $\mu^{+}$SR data were analyzed using the program musrfit [86].
[1] H. Kageyama, K. Hayashi, K. Maeda, J. P. Attfield, Z. Hiroi, J. M. Rondinelli, and K. R. Poeppelmeier, Expanding frontiers in materials chemistry and physics with multiple anions, Nat. Commun. 9, 772 (2018). 
[2] F. Nafezarefi, H. Schreuders, B. Dam, and S. Cornelius, Photochromism of rare-earth metal-oxy-hydrides, Appl. Phys. Lett. 111, 103903 (2017).

[3] F. Nafezarefi, S. Cornelius, J. Nijskens, H. Schreuders, and B. Dam, Effect of the addition of zirconium on the photochromic properties of yttrium oxy-hydride, Sol. Energy Mater. Sol. Cells 200, 109923 (2019).

[4] S. Cornelius, G. Colombi, F. Nafezarefi, H. Schreuders, R. Heller, F. Munnik, and B. Dam, Oxyhydride nature of rareearth-based photochromic thin films, J. Phys. Chem. Lett. 10, 1342 (2019).

[5] G. Colombi, T. de Krom, D. Chaykina, S. Eijt, S. Cornelius, and B. Dam, Influence of cation $(\mathrm{RE}=\mathrm{Gd}, \mathrm{Y}, \mathrm{Sc})$ and $\mathrm{O}: \mathrm{H}$ anion ratio on the photochromic properties of $\mathrm{REO}_{x} \mathrm{H}_{3-2 x}$ thin films, ACS Photonics 8, 709 (2021).

[6] K. Fukui, S. Iimura, T. Tada, S. Fujitsu, M. Sasase, H. Tamatsukuri, T. Honda, K. Ikeda, T. Otomo, and H. Hosono, Characteristic fast $\mathrm{H}^{-}$ion conduction in oxygen-substituted lanthanum hydride, Nat. Commun. 10, 2578 (2019).

[7] H. Ubukata, T. Broux, F. Takeiri, K. Shitara, H. Yamashita, A. Kuwabara, G. Kobayashi, and H. Kageyama, Hydride conductivity in an anion-ordered fluorite structure LnHO with an enlarged bottleneck, Chem. Mater. 31, 7360 (2019).

[8] T. Mongstad, C. Platzer-Björkman, J. P. Maehlen, L. P. A. Mooij, Y. Pivak, B. Dam, E. S. Marstein, B. C. Hauback, and S. Z. Karazhanov, A new thin film photochromic material: Oxygen-containing yttrium hydride, Sol. Energy Mater. Sol. Cells 95, 3596 (2011).

[9] R. Araujo, Inorganic photochromic systems, Mol. Cryst. Liq. Cryst. Sci. Technol., Sect. A 297, 1 (2006).

[10] D. Caurant, D. Gourier, D. Vivien, and M. Prassas, Bleaching mechanism of silver halide photochromic glasses, J. Appl. Phys. 73, 1657 (1993).

[11] C. V. Chandran, H. Schreuders, B. Dam, J. W. G. Janssen, J. Bart, A. P. M. Kentgens, and P. J. M. van Bentum, Solidstate NMR studies of the photochromic effects of thin films of oxygen-containing yttrium hydride, J. Phys. Chem. C 118, 22935 (2014).

[12] S. K. Deb and L. J. Forrestal, Photochromism in inorganic systems, in Photochromism, edited by G. H. Brown, Techniques of Chemistry, Vol. 3 (Wiley-Interscience, 1971), pp. 633-665.

[13] M. Månsson and J. Sugiyama, Muon-spin relaxation study on Li- and Na-diffusion in solids, Phys. Scr. 88, 068509 (2013).

[14] J. Sugiyama, I. Umegaki, M. Matsumoto, K. Miwa, H. Nozaki, Y. Higuchi, T. Noritake, O. K. Forslund, M. Månsson, S. P. Cottrell, A. Koda, E. J. Ansaldo, and J. H. Brewer, Desorption reaction in $\mathrm{MgH}_{2}$ studied with in-situ $\mu^{+} \mathrm{SR}$, Sustainable Energy Fuels 3, 956 (2019).

[15] T. U. Ito, A. Koda, K. Shimomura, W. Higemoto, T. Matsuzaki, Y. Kobayashi, and H. Kageyama, Excited configurations of hydrogen in the $\mathrm{BaTiO}_{3-x} \mathrm{H}_{x}$ perovskite lattice associated with hydrogen exchange and transport, Phys. Rev. B 95, 020301(R) (2017).

[16] R. C. Vilão, R. B. L. Vieira, H. V. Alberto, J. M. Gil, and A. Weidinger, Role of the transition state in muon implantation, Phys. Rev. B 96, 195205 (2017).

[17] J. N. Huiberts, R. Griessen, J. H. Rector, R. J. Wijngaarden, J. P. Dekker, D. G. de Groot, and N. J. Koeman, Yttrium and lanthanum hydride films with switchable optical properties, Nature (London) 380, 231 (1996).
[18] See Supplemental Material at http://link.aps.org/supplemental/ 10.1103/PhysRevB.103.224106 for optical transmission data, details of the $\mathrm{YH}_{2-\delta} \mathrm{ZF}$ fitting, fitted TF and $\mathrm{ZF}$ spectra for yttrium oxyhydrides, behavior of $\lambda_{\mathrm{TF}}$ under illumination, and reversibility of $A_{\mathrm{TF}, \mathrm{dia}}$ with temperature.

[19] T. Prokscha, E. Morenzoni, K. Deiters, F. Foroughi, D. George, R. Kobler, A. Suter, and V. Vrankovic, The new beam at PSI: A hybrid-type large acceptance channel for the generation of a high intensity surface-muon beam, Nucl. Instrum. Methods Phys. Res., Sect. A 595, 317 (2008).

[20] E. Morenzoni, H. Gluckler, T. Prokscha, R. Khasanov, H. Luetkens, M. Birke, E. M. Forgan, C. Niedermayer, and M. Pleines, Implantation studies of $\mathrm{keV}$ positive muons in thin metallic layers, Nucl. Instrum. Methods Phys. Res., Sect. B 192, 254 (2002).

[21] W. Eckstein, Computer Simulation of Ion-Solid Interactions, 1 st ed., Springer Series in Materials Science (Springer, Berlin, 1991).

[22] A. Yaouanc and P. D. de Réotier, Muon Spin Rotation, Relaxation, and Resonance: Applications to Condensed Matter, International Series of Monographs on Physics (Oxford University Press, 2011).

[23] S. J. Blundell, Muon-spin rotation studies of electronic properties of molecular conductors and superconductors, Chem. Rev. 104, 5717 (2004).

[24] B. D. Patterson, Muonium states in semiconductors, Rev. Mod. Phys. 60, 69 (1988).

[25] V. G. Storchak, O. E. Parfenov, J. H. Brewer, P. L. Russo, S. L. Stubbs, R. L. Lichti, D. G. Eshchenko, E. Morenzoni, V. P. Zlomanov, A. A. Vinokurov, and V. G. Bamburov, Novel muonium centers-magnetic polarons-in magnetic semiconductors, Phys. B: Condens. Matter 404, 899 (2009).

[26] V. Storchak, S. F. J. Cox, S. P. Cottrell, J. H. Brewer, G. D. Morris, D. J. Arseneau, and B. Hitti, Muonium Formation via Electron Transport in Silicon, Phys. Rev. Lett. 78, 2835 (1997).

[27] D. C. Walker, Muonium: A light isotope of hydrogen, J. Phys. Chem. 85, 3960 (1981).

[28] W. J. Kossler, H. E. Schone, J. R. Kempton, B. Hitti, C. E. Stronach, G. A. Styles, and E. F. W. Seymour, A comparative muon spin relaxation ( $\mu$ SR) study of titanium and yttrium dihydrides, J. Less-Common Met. 129, 327 (1987).

[29] J. Sugiyama, Spin polarized beam for battery materials research: $\mu \pm \mathrm{SR}$ and $\beta$-NMR, Hyperfine Interact. 240, 17 (2019).

[30] L. M. Cameron and C. A. Sholl, Transverse field depolarization rates for muons in metals, J. Less-Common Met. 172-174, 762 (1991).

[31] O. Hartmann, S. W. Harris, R. Wappling, and R. Hempelmann, Challenging muon diffusion studies in F.C.C. metals, Phys. Scr. 45, 402 (1992).

[32] H. V. Alberto, R. C. Vilão, J. M. Gil, J. P. Duarte, R. B. L. Vieira, A. Weidinger, J. P. Leitão, A. F. d. Cunha, M. G. Sousa, J. P. Teixeira, P. A. Fernandes, P. M. P. Salomé, K. Timmo, M. Loorits, A. Amato, H. Luetkens, T. Prokscha, A. Suter, and Z. Salman, Muonium states in $\mathrm{Cu}_{2} \mathrm{ZnSnS}_{4}$ solar cell material, J. Phys.: Conf. Ser. 551, 012045 (2014).

[33] I. McKenzie, Z. Salman, S. R. Giblin, Y. Y. Han, G. W. Leach, E. Morenzoni, T. Prokscha, and A. Suter, Polymer dynamics near the surface and in the bulk of poly(tetrafluoroethylene) 
probed by zero-field muon-spin-relaxation spectroscopy, Phys. Rev. E 89, 022605 (2014).

[34] T. Lancaster, S. J. Blundell, P. J. Baker, M. L. Brooks, W. Hayes, F. L. Pratt, J. L. Manson, M. M. Conner, and J. A. Schlueter, Muon-Fluorine Entangled States in Molecular Magnets, Phys. Rev. Lett. 99, 267601 (2007).

[35] J. H. Brewer, S. R. Kreitzman, D. R. Noakes, E. J. Ansaldo, D. R. Harshman, and R. Keitel, Observation of muon-fluorine "hydrogen bonding" in ionic crystals, Phys. Rev. B: Condens. Matter 33, 7813 (1986).

[36] The diamagnetic character of the depolarization signal shows that the muon is in either the $\mathrm{Mu}^{+}$or $\mathrm{Mu}^{-}$state. However, the short $\mathrm{H}-\mu$ distance points to a strong binding of the muon to a neighboring $\mathrm{H}^{-}$, indicating that the muon is positively charged. Moreover, for the muon to accommodate two electrons in a $\mathrm{Mu}^{-}$state, the muon would need to reside in an interstitial site like the $\mathrm{H}^{-}$, which is excluded in view of the observed short $\mathrm{H}-\mu$ distance. Lastly, it is unlikely for a $\mathrm{Mu}^{-}$to replace $\mathrm{a} \mathrm{H}^{-}$ due to its light mass. Thus, although $\mathrm{Mu}^{-}$may be present in this material, the vast majority $(\sim 75 \%)$ of the muons behave as $\mathrm{Mu}^{+}$ and are described well by the $\mathrm{H}-\mu$ spin-entangled complex.

[37] J. N. Huiberts, R. Griessen, R. J. Wijngaarden, M. Kremers, and C. Van Haesendonck, Logarithmic Divergence of the Electrical Resistivity in the Metal Hydride $\mathrm{YH}_{3-\delta}$, Phys. Rev. Lett. 79, 3724 (1997).

[38] P. Ngene, T. Radeva, M. Slaman, R. J. Westerwaal, H. Schreuders, and B. Dam, Seeing hydrogen in colors: Low-cost and highly sensitive eye readable hydrogen detectors, Adv. Funct. Mater. 24, 2374 (2014).

[39] P. Ngene, T. Radeva, R. Westerwaal, H. Schreuders, and B. Dam, Eye readable metal hydride based hydrogen tape sensor for health applications, Proc. SPIE 9202, 920203 (2014).

[40] D. Khatamian and F. D. Manchester, The H-Y (hydrogenyttrium) system, Bull. Alloy Phase Diagrams 9, 252 (1988).

[41] G. Majer, J. Gottwald, D. T. Peterson, and R. G. Barnes, Model-independent measurements of hydrogen diffusivity in the yttrium dihydrides, J. Alloys Compd. 330-332, 438 (2002).

[42] M. Di Vece and J. J. Kelly, Electrochemical study of hydrogen diffusion in yttrium hydride switchable mirrors, J. Alloys Compd. 356-357, 156 (2003).

[43] R. Goc, O. J. Zogal, A. H. Vuorimaki, and E. E. Ylinen, Van Vleck second moments and hydrogen diffusion in $\mathrm{YH}_{2.1}$ : Measurements and simulations, Solid State Nucl. Magn. Reson. 25, 133 (2004).

[44] J. N. Daou and P. Vajda, Hydrogen ordering and metalsemiconductor transitions in the system $\mathrm{YH}_{2 \pm \delta}$, Phys. Rev. B 45, 10907 (1992).

[45] J. Sugiyama, O. K. Forslund, E. Nocerino, N. Matsubara, K. Papadopoulos, Y. Sassa, S. P. Cottrell, A. D. Hillier, K. Ishida, M. Månsson, and J. H. Brewer, Lithium diffusion in $\mathrm{LiMnPO}_{4}$ detected with $\mu^{ \pm}$SR, Phys. Rev. Res. 2, 033161 (2020).

[46] T. R. Adams, R. L. Lichti, and T. L. Gibson, Electric-field gradient at oxygen sites in RCBO: Effects from a positive muon, Hyperfine Interact. 86, 561 (1994).

[47] J. Sugiyama, K. Mukai, Y. Ikedo, H. Nozaki, M. Mansson, and I. Watanabe, Li Diffusion in $\mathrm{Li}_{x} \mathrm{CoO}_{2}$ Probed by Muon-Spin Spectroscopy, Phys. Rev. Lett. 103, 147601 (2009).

[48] M. Hiraishi, K. M. Kojima, H. Okabe, S. Takeshita, A. Koda, R. Kadono, R. Khasanov, S. Iimura, S. Matsuishi, and H. Hosono, Magnetism driven by strong electronic correlations in the heavily carrier-doped iron oxypnictide $\mathrm{LaFeAsO}_{0.49} \mathrm{H}_{0.51}$, Phys. Rev. B 101, 174414 (2020).

[49] P. Dalmas de Réotier and A. Yaouanc, Muon spin rotation and relaxation in magnetic materials, J. Phys.: Condens. Matter 9, 9113 (1997).

[50] T. Prokscha, H. Luetkens, E. Morenzoni, G. J. Nieuwenhuys, A. Suter, M. Dobeli, M. Horisberger, and E. Pomjakushina, Depth dependence of the ionization energy of shallow hydrogen states in $\mathrm{ZnO}$ and CdS, Phys. Rev. B 90, 235303 (2014).

[51] T. U. Ito, W. Higemoto, T. D. Matsuda, A. Koda, and K. Shimomura, Shallow donor level associated with hydrogen impurities in undoped $\mathrm{BaTiO}_{3}$, Appl. Phys. Lett. 103, 042905 (2013).

[52] R. C. Vilão, J. M. Gil, A. Weidinger, H. V. Alberto, J. Piroto Duarte, N. Ayres de Campos, R. L. Lichti, K. H. Chow, S. P. Cottrell, and S. F. J. Cox, Acceptor level of interstitial muonium in ZnSe and ZnS, Phys. Rev. B 77, 235212 (2008).

[53] V. G. Storchak, D. G. Eshchenko, J. H. Brewer, S. P. Cottrell, and R. L. Lichti, Muonium in InSb: Shallow acceptor versus deep trap or recombination center, Phys. Rev. B 73, 081203(R) (2006).

[54] A. Miniotas, B. Hjörvarsson, L. Douysset, and P. Nostell, Gigantic resistivity and band gap changes in $\mathrm{GdO}_{y} \mathrm{H}_{x}$ thin films, Appl. Phys. Lett. 76, 2056 (2000).

[55] R. C. Vilão, R. B. L. Vieira, H. V. Alberto, J. M. Gil, A. Weidinger, R. L. Lichti, P. W. Mengyan, B. B. Baker, and J. S. Lord, Barrier model in muon implantation and application to $\mathrm{Lu}_{2} \mathrm{O}_{3}$, Phys. Rev. B 98, 115201 (2018).

[56] R. C. Vilão, R. B. L. Vieira, H. V. Alberto, J. M. Gil, A. Weidinger, R. L. Lichti, B. B. Baker, P. W. Mengyan, and J. S. Lord, Muonium donor in rutile $\mathrm{TiO}_{2}$ and comparison with hydrogen, Phys. Rev. B 92, 081202(R) (2015).

[57] R. B. L. Vieira, R. C. Vilão, A. G. Marinopoulos, P. M. Gordo, J. A. Paixão, H. V. Alberto, J. M. Gil, A. Weidinger, R. L. Lichti, B. Baker, P. W. Mengyan, and J. S. Lord, Isolated hydrogen configurations in zirconia as seen by muon spin spectroscopy and ab initio calculations, Phys. Rev. B 94, 115207 (2016).

[58] A. Rabis, T. Prokscha, E. Fabbri, Z. Salman, T. Schmidt, and A. Suter, Investigation of hydrogen-like muonium states in $\mathrm{Nb}$ doped $\mathrm{SnO}_{2}$ films, JPS Conf. Proc. 21, 011033 (2018).

[59] H. V. Alberto, R. C. Vilão, R. B. L. Vieira, J. M. Gil, A. Weidinger, M. G. Sousa, J. P. Teixeira, A. F. da Cunha, J. P. Leitão, P. M. P. Salomé, P. A. Fernandes, T. Törndahl, T. Prokscha, A. Suter, and Z. Salman, Slow-muon study of quaternary solar-cell materials: Single layers and $p-n$ junctions, Phys. Rev. Mater. 2, 025402 (2018).

[60] G. Colombi, S. Cornelius, A. Longo, and B. Dam, Structure model for anion-disordered photochromic Gd oxyhydride thin films, J. Phys. Chem. C 124, 13541 (2020).

[61] E. L. Silva, A. G. Marinopoulos, R. C. Vilao, R. B. L. Vieira, H. V. Alberto, J. Piroto Duarte, and J. M. Gil, Hydrogen impurity in yttria: $A b$ initio and $\mu$ SR perspectives, Phys. Rev. B 85, 165211 (2012).

[62] E. L. da Silva, A. G. Marinopoulos, R. B. L. Vieira, R. C. Vilão, H. V. Alberto, J. M. Gil, R. L. Lichti, P. W. Mengyan, and B. B. Baker, Electronic structure of interstitial hydrogen in lutetium oxide from $\mathrm{DFT}+U$ calculations and comparison study with $\mu$ SR spectroscopy, Phys. Rev. B 94, 014104 (2016). 
[63] N. B. Mikheev and I. A. Rumer, Stabilization of the divalent state for the lanthanides and actinides in solutions, melts and clusters, Radiochimica Acta 85, 49 (1999).

[64] G. Kobayashi, Y. Hinuma, S. Matsuoka, A. Watanabe, M. Iqbal, M. Hirayama, M. Yonemura, T. Kamiyama, I. Tanaka, and R. Kanno, Pure $\mathrm{H}^{-}$conduction in oxyhydrides, Science 351, 1314 (2016).

[65] S. D. Cohen and G. A. Newman, Inorganic photochromism, J. Photogr. Sci. 15, 290 (1967).

[66] D. L. Staebler and S. E. Schnatterly, Optical studies of a photochromic color center in rare-earth-doped $\mathrm{CaF}_{2}$, Phys. Rev. B 3, 516 (1971).

[67] K. Yokoyama, P. Murahari, P. Heathcote, L. Nuccio, J. S. Lord, N. A. Morley, and A. J. Drew, Future directions of $\mu$ SR-laser excitation, Phys. Scr. 88, 068511 (2013).

[68] K. Shimomura, P. Bakule, F. L. Pratt, K. Ishida, K. Ohishi, I. Watanabe, Y. Matsuda, K. Nagamine, E. Torikai, and K. Nishiyama, Photo detachment of negatively charged muonium in GaAs by laser irradiation, Phys. Procedia 30, 224 (2012).

[69] T. Prokscha, K. H. Chow, Z. Salman, E. Stilp, and A. Suter, Direct Observation of Hole Carrier-Density Profiles and Their Light-Induced Manipulation at the Surface of Ge, Phys. Rev. Appl. 14, 014098 (2020).

[70] T. Prokscha, K. H. Chow, E. Stilp, A. Suter, H. Luetkens, E. Morenzoni, G. J. Nieuwenhuys, Z. Salman, and R. Scheuermann, Photo-induced persistent inversion of germanium in a 200-nm-deep surface region, Sci. Rep. 3, 2569 (2013).

[71] J. Montero, F. A. Martinsen, M. García-Tecedor, S. Z. Karazhanov, D. Maestre, B. Hauback, and E. S. Marstein, Photochromic mechanism in oxygen-containing yttrium hydride thin films: An optical perspective, Phys. Rev. B 95, 201301(R) (2017).

[72] J. Montero and S. Z. Karazhanov, Spectroscopic ellipsometry and microstructure characterization of photochromic oxygencontaining yttrium hydride thin films, Phys. Status Solidi A 215, 1701039 (2018).

[73] E. M. Baba, J. Montero, E. Strugovshchikov, E. O. Zayim, and S. Karazhanov, Light-induced breathing in photochromic yttrium oxy-hydrides, Phys. Rev. Mater. 4, 025201 (2020).
[74] B. Singh, S. Ghosh, S. Aich, and B. Roy, Low temperature solid oxide electrolytes (LT-SOE): A review, J. Power Sources 339, 103 (2017).

[75] X. Liu, T. S. Bjørheim, and R. Haugsrud, Formation of defects and their effects on hydride ion transport properties in a series of $\mathrm{K}_{2} \mathrm{NiF}_{4}$-type oxyhydrides, J. Mater. Chem. A 6, 1454 (2018).

[76] O. S. Fjellvag, J. Armstrong, P. Vajeeston, and A. O. Sjastad, New insights into hydride bonding, dynamics, and migration in $\mathrm{La}_{2} \mathrm{LiHO}_{3}$ oxyhydride, J. Phys. Chem. Lett. 9, 353 (2018).

[77] F. J. A. den Broeder, S. J. van der Molen, M. Kremers, J. N. Huiberts, D. G. Nagengast, A. T. M. van Gogh, W. H. Huisman, N. J. Koeman, N. I. Koeman, B. Dam, J. H. Rector, S. Plota, M. Haaksma, R. M. N. Hanzen, R. M. Jungblut, P. A. Duine, and R. Griessen, Visualization of hydrogen migration in solids using switchable mirrors, Nature (London) 394, 656 (1998).

[78] K. Hayashi, S. Matsuishi, T. Kamiya, M. Hirano, and H. Hosono, Light-induced conversion of an insulating refractory oxide into a persistent electronic conductor, Nature (London) 419, 462 (2002).

[79] K. Hayashi, Kinetics of electron decay in hydride ion-doped mayenite, J. Phys. Chem. C 115, 11003 (2011).

[80] K. Hayashi, P. V. Sushko, Y. Hashimoto, A. L. Shluger, and H. Hosono, Hydride ions in oxide hosts hidden by hydroxide ions, Nat. Commun. 5, 3515 (2014).

[81] K. Hayashi and H. Hosono, Green apatites: Hydride ions, electrons and their interconversion in the crystallographic channel, Phys. Chem. Chem. Phys. 18, 8186 (2016).

[82] J. Chai, Z. Shao, H. Wang, C. Ming, W. Oh, T. Ye, Y. Zhang, X. Cao, P. Jin, S. Zhang, and Y.-Y. Sun, Ultrafast processes in photochromic material $\mathrm{YH}_{x} \mathrm{O}_{y}$ studied by excited-state density functional theory simulation, Sci. China Mater. 63, 1579 (2020).

[83] T. He and J. Yao, Photochromism of molybdenum oxide, J. Photochem. Photobiol. C: Photochem. Rev. 4, 125 (2003).

[84] H. Bill and G. Calas, Color centers, associated rare-earth ions and the origin of coloration in natural fluorites, Phys. Chem. Miner. 3, 117 (1978).

[85] J. Tennyson and S. Miller, Fractionation effects in muoniummolecular hydrogen reactions, J. Chem. Phys. 90, 2524 (1989).

[86] A. Suter and B. M. Wojek, Musrfit: A free platformindependent framework for $\mu \mathrm{SR}$ data analysis, Phys. Procedia 30, 69 (2012). 